Check for updates

Cite this: J. Mater. Chem. B, 2022, 10,1734

Received 8th December 2021, Accepted 31st January 2022

DOI: $10.1039 / \mathrm{d} 1 \mathrm{tb} 02706 \mathrm{a}$

rsc.li/materials-b

\title{
Engineering strategies to achieve efficient in vitro expansion of haematopoietic stem cells: development and improvement
}

\author{
Bangheng Liu, ${ }^{\text {ab }}$ Chao Tao, ${ }^{b}$ Zhonglian Wu, ${ }^{c}$ Hang Yao*c and Dong-An Wang (D) *ab \\ Haematopoietic stem cells are the basis for building and maintaining lifelong haematopoietic \\ mechanisms and an important resource for the treatment of blood disorders. Haematopoietic niches \\ are a microenvironment in the body where stem cells tend to accumulate, with some nurse cells \\ protecting and regulating stem cells. On the basis of biology, materials science, and engineering, \\ researchers have constructed stem cell niches to address the current clinical shortage of stem cells \\ and to explore stem cell behaviour for biomedical research. Herein, three main resource categories \\ involved in haematopoietic stem cell niche engineering are reviewed: first, the basic approach to \\ construct bionic cell culture environments is to use cytokines, nurse cells or extracellular matrix; \\ second, microscale technologies are applied to mimic the properties of natural stem cell niches; and \\ finally, biomaterials are used to construct the three-dimensional extracellular matrix-like culture \\ environment.
}

\section{Introduction}

Haematopoietic stem cells (HSCs) are rare multipotent stem cells with self-renewal ability that can constantly maintain the homeostasis of mature blood cells and quickly differentiate to replenish immune cells in the case of inflammation and/or injury. ${ }^{1-3}$ The key to this process is the self-renewal and differentiation of HSCs. ${ }^{4}$ It is demonstrated that HSCs grow in a niche, a specific microenvironment that regulates their growth and proliferation. ${ }^{5,6}$ The HSC niche accommodates cellular, chemical and physical components surrounding HSCs, influencing their synergy and signal transmission stimuli received from support cells. ${ }^{7}$

The HSC niche is a principal node for haematopoiesis and blood formation and provides clues for understanding the blood system, oncogenesis and inevitable ageing. ${ }^{4,8}$ From the initial HSCs to mature cells, HSC diversity starts in the HSC niche and then gradually but robustly develops, and is related to the stemness, maintenance of stemness, quiescent state, and unilineage differentiation of HSCs. ${ }^{4-9-11}$ In addition, a prominent induction mechanism in the HSC niche plays a

\footnotetext{
${ }^{a}$ Department of Biomedical Engineering, City University of Hong Kong, 83 Tat Chee Avenue, Kowloon, Hong Kong SAR. E-mail: dwang229@cityu.edu.hk

${ }^{b}$ Karolinska Institutet Ming Wai Lau Centre for Reparative Medicine, HKSTP, Sha Tin, Hong Kong SAR

${ }^{c}$ School of Chemistry and Chemical Engineering, Yangzhou University, Yangzhou 225009, Jiangsu, P. R. China. E-mail: yaohang@yzu.edu.cn
}

guiding role in the study of blood cell cancerogenesis. ${ }^{12-14}$ The HSC niche can also reflect the relationship between ageing and haematopoietic cell regeneration and can be used to explore HSC's potential for rejuvenation (Table 1$).{ }^{15-17}$

In addition to their importance in biomedical research, HSCs are also crucial in clinical use. ${ }^{18,19}$ Currently, the main curative treatment for haematologic and lymphoid cancers, and many other disorders such as autoimmune disorders, amyloidosis and aplastic anaemia, is HSC transplantation (HSCT). ${ }^{18,20}$ However, the shortage of HSCs limits HSCT in clinical applications. Therefore, the ultimate goal of studying the HSC niche is to address the shortage of therapeutic HSC sources for use in blood engraftment. For this purpose, the general solution relies on the efficient and high-quality expansion of HSCs in vitro; that is, the efficient proliferation of abundant therapeutic cells while maintaining the HSC phenotype by preventing differentiation is important. This solution is embodied by an artificial niche engineered for HSCs.

In this review, we focus on the engineering methods to achieve effective HSC amplification in vitro involving three parts. First, the characteristics of the HSC bone marrow niche and then details of the biochemical stimulation strategies based on static culture and 3D culture approaches are introduced. Then the role of microscale technology in advancing the development of HSC artificial niches is reviewed. Finally, the materials used to construct the in vitro culture system and the pros and cons of the culture system construction methods are summarized (Table 2). 
Table 1 Engineering strategies and platforms in response to the characteristics of the HSC niche

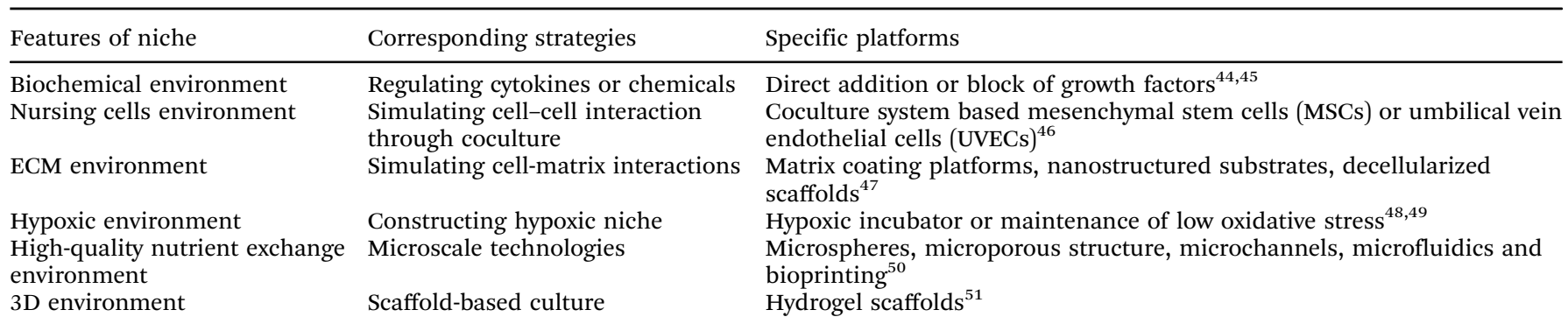

Table 2 The pros and cons of biochemical stimulation and improvement methods

\begin{tabular}{|c|c|c|c|}
\hline Coculture & No additional cytokines required & Difficulties in detecting potential pathways ${ }^{103}$ & Nursing cell encapsulation \\
\hline Hypoxic niche & Closing physiological oxygen conditions & Single factor dominates ${ }^{86}$ & $\begin{array}{l}\text { Combining other } \\
\text { technologies }\end{array}$ \\
\hline
\end{tabular}

\section{Haematopoietic stem cells and their natural niche}

HSCs first appear in the aorta-gonad-mesonephros (AGM) region, and later, with the transfer of haematopoiesis functional sites, HSCs emerge in the foetal liver and mainly participate in circulation in the body. ${ }^{21,22}$ Finally, with the development of the body, the bone marrow becomes the basis of the haematopoiesis system. In addition to this path, the placenta is believed to influence haematopoiesis during the period from AGM initiation to the foetal liver emergence. ${ }^{4}$ Compared to HSCs in the AGM region, HSCs in the placenta tend to differentiate into progenitors. HSCs in the bone marrow are largely quiescent and are mainly involved in the circulatory system, except those in the foetal liver. ${ }^{4}$ Under normal conditions, the behaviours of stem cells are critically regulated by both intrinsic factors and extrinsic cues from the surrounding microenvironment in vivo; these areas of external regulatory signalling are termed stem cell niches. HSCs are mainly located in the bone marrow during adulthood. In bone marrow, human HSCs are located either next to osteoblasts on the endophytic wall of the bone or adjacent to the endothelial cells (ECs) of sinusoidal blood vessels in marrow fluid. ${ }^{23} \mathrm{~A}$ summary of the bone marrow niche is shown in Fig. 1.

It is reported that HSCs were attached to spindle-shaped $\mathrm{N}$-cadherin ${ }^{+} \mathrm{CD}_{4} 5^{-}$osteoblasts (SNO) in the bone niche for a long time and that the number of SNO cells determined the number of HSCs. ${ }^{24}$ Researchers believe that SNO cells may support HSCs through the specific adhesion of N-cadherin and $\beta$ catenin and play critical regulatory roles affecting HSC function through notch activation by generating Jagged $1 .{ }^{7,24} \mathrm{~N}$-cadherin is also the main target of angiopoietin-1 (Ang-1)/Tie-2 signalling,

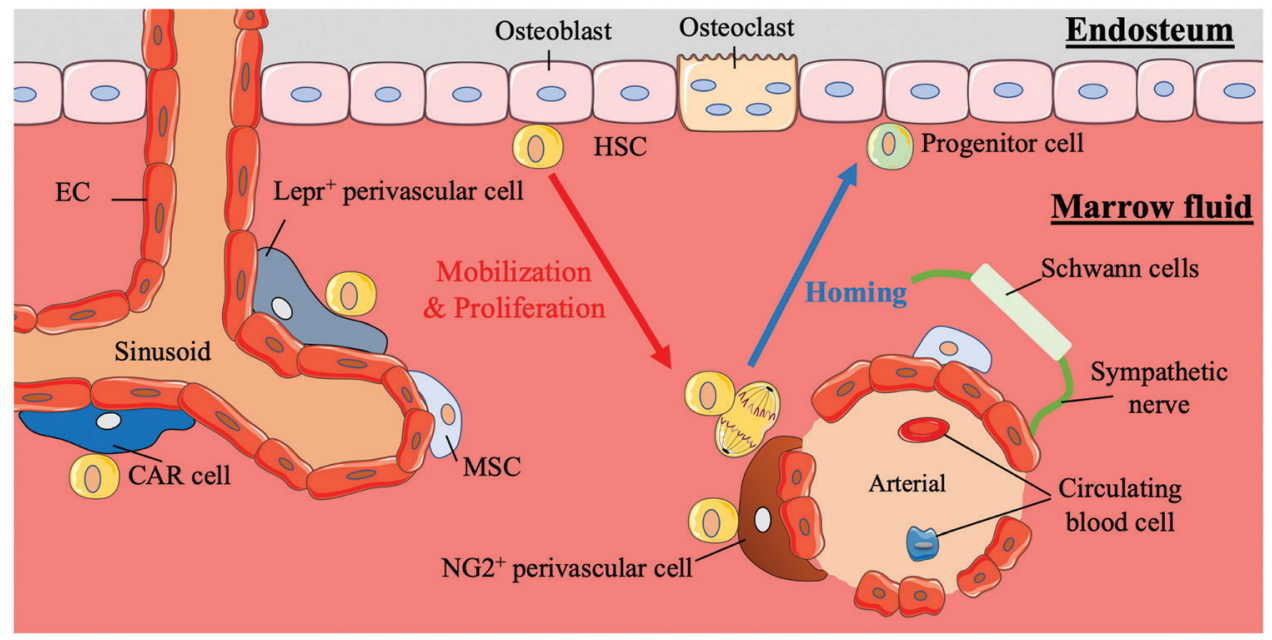

Fig. 1 A sketch of the haematopoietic stem cell bone marrow niche. 
which can facilitate the preservation of HSC quiescence. ${ }^{25}$ SNO cells and the bone cavity create a microenvironment suitable for long-term HSC proliferation while maintaining the HSC phenotype. The bone niche also plays an anchoring role in the homing process of HSCs. Chemokine ligand 12 (CXCL12, also known as stromal cell-derived factor (SDF)-1) secreted by osteoblasts or other nurse cells recruits HSCs to the bone marrow and directs them to their appropriate niche by interacting with the CXCR4 receptor expressed by HSCs. ${ }^{26,27}$

HSCs also gather around blood vessels inside the bone marrow. ${ }^{28}$ This perivascular location near the endosteum is called the vascular niche. ${ }^{29}$ In the vascular niche, stromal cells and endothelial cells surround HSCs and their progeny forming a protective environment and maintaining the HSC population by secreting CXCL12, stem cell factor (SCF, also known as KITL) and probably other cytokines. ${ }^{23,30,31}$ Among these support cells, a particular type of perivascular stromal cell can secrete a large amount of CXCL12 and contact HSCs directly, CXCL2-abundant reticular (CAR) cells, which surround endothelial cells on sinusoids and arterioles or are located near the bone niche of HSCs. ${ }^{32}$ The localization of HSCs in the quiescent and activation state differs. Quiescent HSCs are associated with small arterial regions where neural-glial antigen 2-positive $\left(\mathrm{NG}^{+}\right)$periarteriolar cells are located. In contrast, activated HSCs are clustered near the perisinusoidal region expressing a leptin receptor (Lepr). ${ }^{33}$ Other cells, including macrophages, megakaryocytes, sympathetic nerves, and non-myelinating Schwann cells, also play roles in regulating the vascular niche. ${ }^{34,35}$

The intricate physical and chemical environment inside the bone marrow niche is crucial to the regulation of HSCs. The extracellular matrix (ECM) is a complex system involved in the development of the inner three-dimensional (3D) structure of bone marrow and composed of collagen, proteoglycans, glycoproteins and glycosaminoglycans. ${ }^{36}$ The ECM not only provides structural scaffolding but also regulates stem cell behaviour. Different ECM molecules change the adhesion, proliferation, survival, migration and differentiation of stem cells. ${ }^{37-41}$ Simultaneously, changes in the stiffness of the bone marrow can affect the behaviour of HSCs. Changes in the external physical environment can be detected by stem cells through complex mechanical sensing systems, and modulate the subsequent transcription process. ${ }^{42}$ The influence of changes in oxygen content on HSCs cannot be ignored either. The hypoxic tension in the bone marrow niche is considered to be an important reason for maintaining HSC stemness. ${ }^{43}$ This local heterogeneity with a steep gradient influences the behaviours and fates of HSCs.

The natural HSC niche has complex regulatory behaviours with many biochemical and physical components participating in this process. The simulation and in-depth exploration of this platform are of great significance to biomedicine and also to clinical medicine. Thus, in combination with the properties of the HSC niche, there are responsive engineering strategies to simulate in order to create a culture system more suitable for HSC in vitro amplification and maintenance of the phenotype, which will be elaborated in detail in the following sections.

\section{Biochemical stimulation}

\subsection{Regulating cytokines or chemicals to mimic the bone marrow niche}

The development and growth of HSCs are believed to be regulated by a wide range of biomolecules. Cytokines, including SCF, FMS-related tyrosine kinase 3 ligand (Flt3L), granulocyte colony-stimulating factor (G-CSF), interleukin (IL)-3, IL-6, IL-11 and thrombopoietin (TPO), are known regulators that contribute to quiescence maintenance, self-renewal regulation and migration of HSCs in vivo. ${ }^{452-54}$ The combination of these cytokines can achieve diverse selective amplification of HSCs in an in vitro environment, among which the combination of Flt3L, SCF and TPO has been commonly used. For instance, the number of CD $34^{+}$cells can be increased by $28 \pm 9$-fold in vitro in a 10 days treatment of cultured $\mathrm{CD} 34^{+} / \mathrm{CD} 38^{-}$cells with the combination of the above three cytokines. ${ }^{55}$ However, no matter how these cytokines are combined, the effect on long-term culturing of HSCs in vitro is not significant. ${ }^{56}$ Interestingly, compared to soluble cytokines, the matrix-immobilized form of cytokines shows a better supportive effect on the long-term culture of HSCs in vitro. ${ }^{57}$ Mahadik et al. used acrylate-functionalized polyethylene glycol (PEG) tethers to covalently immobilize SCF and proved that the effect of covalent SCF on cell phenotype maintenance is stronger than that of continuous SCF. ${ }^{58}$ Immobilized cytokines such as cell-surface SCF and cell-surface CSF-1 were proven to play supporting roles in the long-term culture of HSCs in vitro by transduction technology according to Friel et al. ${ }^{59}$

Other cytokines have different regulatory effects on HSCs and haematopoietic progenitor cells (HPCs). Tumour necrosis factor-alpha (TNF- $\alpha$ ) not only induces the apoptosis of myeloid progenitor cells but also promotes HSC survival and bone marrow differentiation by activating p65 nuclear factor $\kappa \mathrm{B}$ (NF-KB)-dependent gene programmes. ${ }^{60,61}$ This specificity can prevent necroptosis and induce immune regulation. However, whether TNF- $\alpha$ itself or other ligands induced by TNF- $\alpha$ directly act on HSCs to exert the aforementioned protective function remains to be determined. ${ }^{60}$

Transforming growth factor beta (TGF- $\beta$ ) is one of the most effective inhibitors restricting the growth of HSCs in vitro and is also considered to be the main cytokine maintaining the quiescent state of HSCs. ${ }^{62}$ TGF- $\beta$ not only independently controls the cell cycle of HSCs through the upregulation of $\mathrm{p} 57^{\mathrm{Kip} 2}$ but also inhibits the proliferation of HCSs by changing the expression of cytokine receptors and upregulating inhibitors of cyclin-dependent kinases. ${ }^{44}$ Akhkand et al. blocked the expression of TGF- $\beta$ receptor II on $\mathrm{CD} 34^{+}$cells to increase the expansion rate of $\mathrm{CD} 34^{+}$cells. $^{63}$ In a culture environment with only growth factors, the expansion factor of transfected CD $34^{+}$ cells measured on the eighth day was 28.5 fold, while the expansion factor of $\mathrm{CD} 34^{+}$cells was only 10.8 fold. $^{63}$

GSK3 inhibits Wnt/ $\beta$-catenin signal transduction through the degradation of $\beta$-catenin triggered by phosphorylation and inhibits the expansion of HSCs in vitro. However, interestingly, GSK3 inhibitors can induce $\beta$-catenin activity to promote HSC proliferation and in turn stimulate bone marrow cells to 
produce inflammatory cytokines, which can reduce HSC proliferation by inducing p38 activation. ${ }^{64} \mathrm{Li}$ et al. directly blocked the GSK3 signalling pathways. In impure HSC populations, the number of $\mathrm{CD}_{3}{ }^{+}$cells in the blocking environment was significantly increased compared with that in the unblocked environment, with a 9- to 11 -fold difference after 12 days. ${ }^{64}$

In addition to cytokines, synthetic chemical molecules can be applied to replace specific components of the culture medium. Polyvinyl alcohol (PVA) can replace serum albumin for the culture of HSCs. ${ }^{65}$ Through optimized SCF and TPO concentrations and the use of a fibronectin matrix, this albumin-free in vitro culture method can support the long-term expansion of mouse HSCs for more than 2 months. ${ }^{19}$ However, the albumin-free in vitro culture method for human HSCs needs further improvement. ${ }^{66}$

The biochemical environment of HSCs in the niche is complicated; therefore, to simplify the model and/or to confirm the expression of specific cytokines, conditioned medium is often used for in vitro cultures of HSCs. Breems et al. utilized culture medium in which stromal cells were cultured to promote the proliferation of HSCs. ${ }^{67}$ This method is similar to that of coculturing, which will be introduced in Section 3.2. Moreover, although the blockade of unwanted cytokines needs to be taken into consideration, it increases the uncertainty of the niche culture components.

\subsection{Simulating cell-cell interaction through coculture}

HSCs make close intercellular connections with nurse cells in the bone marrow niche. The physical and chemical mechanisms of the interactions between these cells are still not fully understood. Coculture of these cells is the basis of an in vitro engineering model in which an HSC niche is simulated but not particularly similar to a natural niche. However, using nurse cells as the feeder cells or feeder layer of HSCs for coculture is a direct and simple biochemical bionic means.

In the bone marrow niche, MSCs interact with HSCs and their progenitor cells to form a symbiotic environment, protect the phenotype of the HSCs, and promote HSC self-renewal. MSCs secrete SCF, Flt3L, IL-6, and CSF-1 cytokines, which can promote the expansion of HSCs in vitro and support long-term culture. ${ }^{68}$ Under the same cytokine conditions in vitro, the expansion of $\mathrm{CD} 34^{+} / \mathrm{CD} 38^{-}$cells in a population with MSCs as feeder cells was 45-fold greater after 10 days than that of a group without MSCs as feeder cells. ${ }^{55}$

Genetically engineered MSCs can be used as feeder cells to improve the efficiency of in vitro HSC amplification. As a cytokine that regulates the mobilization, homing and expansion of HSCs, stromal cell-derived factor-1 (SDF-1) can regulate the cell cycle of HSCs by combining with SCF or TPO and thus enhance the survival of HSCs in vitro. ${ }^{69}$ Therefore, Ajami et al. used transfection technology to prepare MSCs capable of overexpressing SDF-1 and SCF (the soluble form and membrane form, respectively). The results showed that the maximum expansion factor of $\mathrm{CD} 34^{+}$cells reached $4.73 \pm 0.26$-fold after seven days. ${ }^{70}$
UVECs can also be used as feeder layers to assist the expansion of HSCs in vitro. UVECs express some of the same surface markers and transcription molecules as HSCs, such as CD34, CD31, Runx1 and GATA-2, to support the expansion and differentiation of HSCs in vitro. ${ }^{71,72}$ Therefore, Li et al. cocultured UVECs as feeder cells with HSCs in a culture medium containing SCF, TPO and Flt3L and tested the HSC stemness and HSC in vitro expansion. The results showed that the feeder-based coculture further promoted the expansion of the HSCs. ${ }^{72}$ Huang et al. also used the cocultivation model of UVECs and HSCs to construct a comprehensive and balanced pool of long-term HSC cultures which effectively maintained the HSC phenotype in vitro. ${ }^{46}$

There are two ways of coculturing HSC: direct coculture and indirect coculture. ${ }^{73}$ In the direct method, cells are in direct contact or adhere together. This method is simple with no technical barriers but is impossible to evaluate the degree of the specific cell interaction. The indirect method involves separating cells in the same culture space through physical isolation methods such as semipermeable membranes. This method avoids labelling cell populations to detect the effects of soluble cytokines, but is not suitable for studies in which physical contact triggers induction.

\subsection{Simulating cell-matrix interactions by matrix coating platforms and nanostructured substrates}

Inspired by the natural niche of HSCs, artificial niches (such as collagen fibres and fibronectin fibrils) were constructed to mimic and engineer the nano-structured ECM that supports cell behaviour on the basis of their own morphology and mechanical properties. HSCs can be induced by changes in substrate elasticity, morphology, and ligand lateral spacing in the culture environment. ${ }^{74,75}$ Instead of using growth factors and cytokines to induce HSCs, the physical characteristics of the material such as substrate elasticity and substrate nanostructures is adjusted to stimulate HSCs and promote in vitro HSC expansion.

A platform based on tropoelastin-coated substrates first used by Holst et al. showed that matrix elasticity and tensile integrity influenced the growth of HSCs. ${ }^{76}$ Choi et al. tested a type I collagen- and fibronectin-functionalized polyacrylamide surface, and photo-crosslinked laminin by sulfo-SANPAH and found that the fibronectin maintained the population of HSCs, the collagen promoted the proliferation of HSCs, and the laminin promoted the differentiation of HSCs into a red blood cell lineage. ${ }^{77}$ Changes in the morphology of the substrate also affect the fate of stem cells. For example, a microcavity with a diameter no larger than $15 \mu \mathrm{m}$ can contribute to an increase in the number of HSCs. ${ }^{78}$ Nanopillars and nanogratings have also been shown to have an effect on the fate of HSCs. ${ }^{79,80}$

The arginine-glycine-aspartic acid (RGD) peptide is key to the ECM guidance of stem cell adhesion, differentiation and proliferation. ${ }^{47}$ Raic et al. confirmed that RGD contributes to the maintenance of the HSC stemness. ${ }^{81}$ Kratzer et al. used RGD to induce the differentiation of HSCs into $\mathrm{T}$ cells and provided a strategy for artificially inducing $\mathrm{T}$ cells. ${ }^{82,83} \mathrm{RGD}$ is 
commonly used as a functional peptide in hydrogels to improve the supportive ability of hydrogels for the expansion of HSCs. In addition to RGD, the connecting segment (CS)-1 motif of fibronectin has been confirmed to support the proliferation of HSCs. $^{83}$

The nanoscale lateral distance between coupled ligands has been shown to be a factor that mediates the interaction between cells and ECM molecules. ${ }^{6}$ Ligands at a specific distance, which is determined by the type of ligand, facilitate the clustering of integrins to form a signalling complex. For peptide ligands derived from small fibronectin, the critical adhesion distance between HSCs is less than $45 \mathrm{~nm}$. However, fibronectin-derived and OPN-derived protein domains can support long-distance cell adhesion. ${ }^{84}$ Winkler et al. used an adhesive RGD peptide and DLL1 to construct a functional surface and proved that ligand density and clustering are key to promoting the proliferation of HSCs. ${ }^{85}$

Indeed, different artificial niche methods can be combined with each other, exerting a synergistic effect on the promotion of HSC amplification. However, pure matrix coating platforms and nanostructured substrates are flawed as they lack multidimensionality, one key aspect of ECM mimics. As a result, the culture model of cells is not spatially consistent with the natural niche. Therefore, a 3D culture method based on biomaterials has been developed and valued.

\subsection{Constructing a hypoxic niche}

Compared with niches of cells in other tissues, the bone niche of HSCs is a hypoxic environment, which ensures that HSCs stay in a quiescent state with an unchanging phenotype. ${ }^{49,86}$ Quiescent HSCs maintain low oxidative stress to prevent self-depletion or differentiation and a high glycolysis rate to generate energy through anaerobic metabolism, which differs from the metabolism of progenitor cells or differentiated offspring cells. ${ }^{87}$ An important transcription factor in this regulatory pathway, hypoxia-inducible factor-1alpha (HIF-1 $\alpha$ ), affects HSCs through the heat shock protein GRP78 and its ligand Cripto. ${ }^{88}$ Without the expression of HIF- $1 \alpha$, HSCs are released from the quiescent state of the cell cycle, gradually age and lose their transplantation capacity. The excessive stability of HIF- $1 \alpha$ (biallelic loss of von Hippel-Lindau protein) arrests the cell cycle and causes a loss of HSC transplantation capacity. ${ }^{49,89}$

Kiani et al. cocultured MSCs with overexpressed HIF-1 $\alpha$ with HSCs and found that the MSCs effectively contributed to HSC phenotype maintenance and inhibited the differentiation of HSCs. ${ }^{90}$ Capitano et al. reduced the oxygen stress response to restore the number and function of HSCs in aged mice. ${ }^{91}$ Kobayashi et al. created a low-oxygen environment to achieve a long-term (one month) quiescent state for HSCs in vitro. ${ }^{48}$ For the construction of a hypoxic culture environment, researchers mainly use a $\mathrm{CO}_{2} / \mathrm{O}_{2}$ incubator to adjust the culture environment to $5 \% \mathrm{CO}_{2} / 1-5 \% \mathrm{O}_{2}$ or $5 \% \mathrm{CO}_{2} / 95 \% \mathrm{~N}_{2}$ for flushing the HSCs in a sealed culture chamber. ${ }^{48,91,92}$ Research on the hypoxic niche mainly focuses on the maintenance of the in vitro HSC phenotype or the regulation of the resting state. The use of the hypoxic niche to promote the high-quality expansion (maintaining the phenotype while inducing high amplification magnification) of HSCs in vitro needs further study. Fig. 2 shows a schematic diagram of the methods by which biological mimics are used to create an HSC artificial niche.

\subsection{Improvements: scaffold-based system and dynamicisation of biochemical stimuli}

Biomaterials such as collagen, tropoelastin, fibronectin or other ECM-derived materials are always used as substrates to fabricate artificial niches in a facile way. This static suspension cell culture method addresses the initial problems of cell-matrix pathway triggering, ${ }^{76,77,93}$ but unfortunately, fails in the biological and physiological cases and leads to obvious distortions. The growth mode of cells in the static suspension model is different from that in the human body, resulting in obvious differences in morphology, differentiation, cell-cell contact, and cell-matrix contact to those of cells growing under physiological conditions in vivo. As a result, there are difficulties in translating the static suspension model into clinical use or pharmacological and physiological use. The cells in a 3D culture model keep their natural forms and functions and proliferate in the same way as in vivo proliferation. 3D models provide a platform to study the interconnection of cells in a complex system with physiologically relevant physical structures that allow cells to grow in multiple
A. Adding cytokines

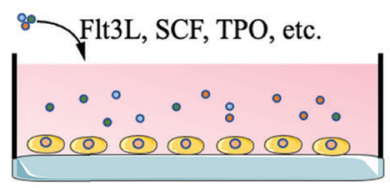

D. Nanostructured substrate

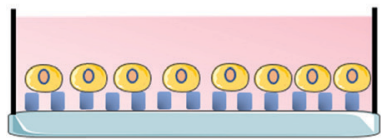

B. Coculture

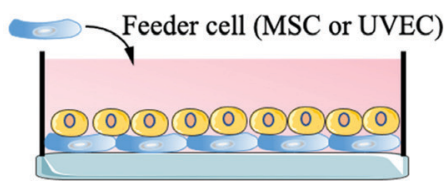

E. Hypoxic niche $\mathrm{CO}_{2} / \mathrm{O}_{2}$ incubator $\left(5 \% \mathrm{CO}_{2} / 1 \% \sim 5 \% \mathrm{O}_{2}\right)$

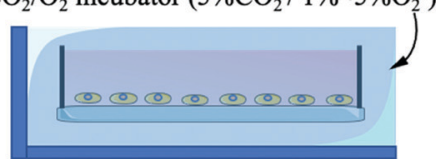

C. Matrix coating

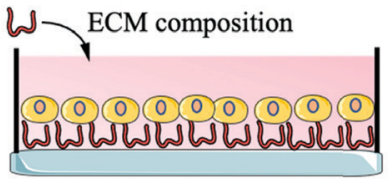

F. Pathway regulatory

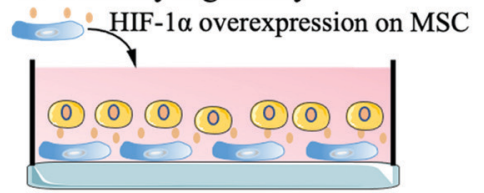

Fig. 2 Biochemical bionics to engineer artificial niches. 
directions. ${ }^{94}$ The 3D model reduces the contact area between the cell and the substrate to maximize the stability of the original morphology of the cell and help maintain the cell function in vitro. HSCs are more active and more responsive to external factors in the $3 \mathrm{D}$ model compared to static suspension models. ${ }^{95}$ A functional 3D model, which avoids the ethical and economic issues, can be a substitute for animal models.

All biochemical stimulations are designed to mimic the original niche characteristics of HSCs and maintain the phenotype and amplification status of HSCs as much as possible. However, this desired outcome can hardly be yielded from in vitro culture of HSCs under traditional cultural conditions via free suspension. ${ }^{96}$ It is hypothesized that this failure is attributed to the irregular and discontinuous dosage and dosing frequency along with the conventional operation of medium change. $^{96,97}$ The development of a 3D culture of HSCs via the traditional method of free suspension is hindered by the inevitable operations of centrifuging and re-suspending steps for medium change every time, which is labour intensive and causes damage to the cultured cells. In contrast, a dynamic 3D culture by means of equipment modulation can address these problems. Medium change can therefore be controlled and performed in a real-time and dynamic manner through programmed automation. In this way, the microfluidic administration of exogenous maintenance additives can better mimic the natural administration in vivo; thus, with the dynamic culture system, proliferation and differentiation resistance can be promoted simultaneously in vitro for quality and efficient expansion of HSCs.

3.5.1 Scaffold-free and scaffold-based systems. 3D culture models can be divided into scaffold-based and scaffold-free systems. A detailed diagram of scaffold-based and scaffold-free systems is shown in Fig. 3.

A scaffold-free system refers to a construct in which cells do not rely on external materials or other cells because they can provide their own self-supporting structures for cell adhesion, growth, and diffusion by forming tissue-like micro-tissue spheres. There are three main categories of scaffold-free systems: non-adhesive surfaces, hanging drops and equipment (spinner flasks or perfusion bioreactors). When culturing on a nonadhesive surface, cells do not attach to the surface but accumulate to form a cell sphere, thus developing a selfassembling 3D culture. In the hanging drop method, the cell culture environment is replaced by hanging drops that promote the self-assembly of cells into microspheres by gravity. The formation of the microstructure ball in the hanging drop method can be easily controlled and highly consistent from batch to batch. Moreover, the hanging drop method can be used to coculture different cell types, ensuring signal exchange between cocultured cells. ${ }^{98}$ In the spinner flask method, a stirrer agitates the culture medium in a flask, which drives the cells into a circular motion and promotes the aggregation of cells, forming microstructures. The perfusion bioreactor system can continuously replenish the new culture fluid while draining the old culture fluid to maintain the optimal growth state of the cells and reduce the accumulation of environmental toxins. ${ }^{99}$ The equipment used in the spinner flask/bioreactor system can improve the cell culture density, quality, and utilization. ${ }^{10,100}$

A scaffold-based system involves a hydrogel scaffold which is generated by natural or synthetic biomaterials and can be further tailored. In addition, biomaterials secreted by cells can be used as scaffold materials, such as decellularized ECM scaffolds or decellularized bone marrow scaffolds. Cells can also be used to construct organoids or microsphere scaffolds to provide an additional anchor for target cells. ${ }^{101}$ The design and use of scaffolds requires consideration of the structure, pore size, morphology, mechanical properties, surface characteristics and degradation products. ${ }^{102}$ Scaffold-based systems have advantages over scaffold-free systems in mimicking the complex and functional ECM environment which is highly relied on for HSC expansion, or controlling the positioning of transplanted cells. ${ }^{95,100}$ Scaffolds provide a controlled environment for cell growth while supporting long-term survival or proliferation of cells in vitro. They are also able to carry growth factors, maintain their protein activity and improve cellular capture of growth regulatory signals. The use of a scaffold-based system can improve the competitive advantage of donor HSCs and increase the success rate of HSCT. ${ }^{33}$
A. Scaffold based (Hydrogel)

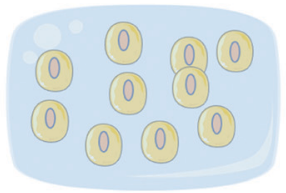

D. Scaffold-free (Hanging drop)

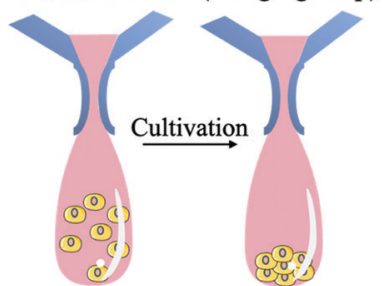

B. Scaffold based (Decellularized materials )

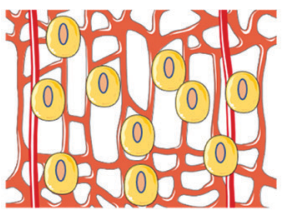

E. Scaffold-free (spinner flask)
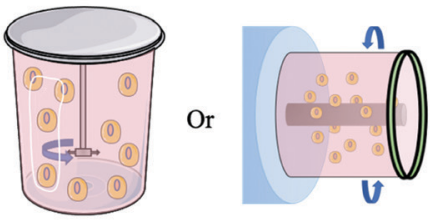

C. Scaffold based (Cell-derived materials )
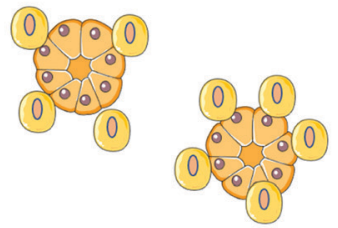

F. Scaffold-free (Perfusion bioreactor)

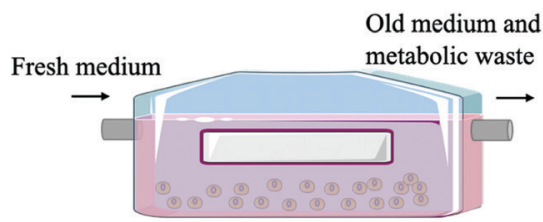

Fig. 3 A sketch of the 3D culture model including scaffold-based and scaffold-free systems. 
3.5.2 Improvement strategies for biochemical stimuli. Cytokines enhance HSC proliferation, but also largely facilitate HSC differentiation at the same time, which is unacceptable for maintaining HSC cell stemness in vitro. ${ }^{103}$ Furthermore, cytokines are unstable in in vitro culture environments and are rapidly inactivated in aqueous liquid, leaving HSCs unable to receive sufficient biological signals to maintain a proliferative or quiescence state. ${ }^{104,105}$ Mhanna et al. then used sulphated scaffolds to improve the capture of cytokines in the culture system. ${ }^{106}$ By sulphating alginate to varying degrees, Mhanna et al. found that an increase in the sulphation degree of hydrogels resulted in an increase in the binding capacity for cell growth factors and maintaining cell stemness. ${ }^{106}$ Comparing heparin, dextran sulfate, $\lambda$-carrageenan, and chondroitin sulfate as binding and stabilizing agents for growth factors, Sun et al. found that sulfated polysaccharide scaffolds helped stabilize cytokines. ${ }^{107}$ This suggests that the sulphated polysaccharide scaffold contributed to maintaining cytokine activity and further cell stemness (Fig. 4). ${ }^{108}$

For coculture, a direct three-dimensional approach is no better than static suspension coculture. Because MSCs or
UVECs can adhere to the dish, they have the advantage of subsequent cell isolation on culture plates. If the HSCs and nursing cells are embedded in the scaffold, subsequent cell isolation or detection would be tedious. ${ }^{109,110}$ The phase separation method is therefore used, i.e. MSCs and HSCs are encapsulated in different hydrogels, which makes the detection and separation easier. ${ }^{111,112}$

In addition, the disadvantages of isolation or characterization can be circumvented by culturing nursing cells into cell spheres and using the size difference between HSCs and cell microspheres. ${ }^{113}$ Hur et al. used mononuclear blood cells to construct cellular microspheres that provided physical support for HSCs and included cytokine modulation such as introduction of SDF-1 and CXCR4. The presence of a large ECM component in the microspheres also greatly facilitated the proliferation of HSCs. $^{114}$ However, heterogeneous cell populations can have other potential effects on growth stimulation and activity maintenance. ${ }^{115}$ Furthermore, potential signalling pathways are difficult to detect and fully demonstrate in the laboratory; therefore, using cells present in the natural HSC niche as microsphere scaffolds is effective in preventing the effects of too many

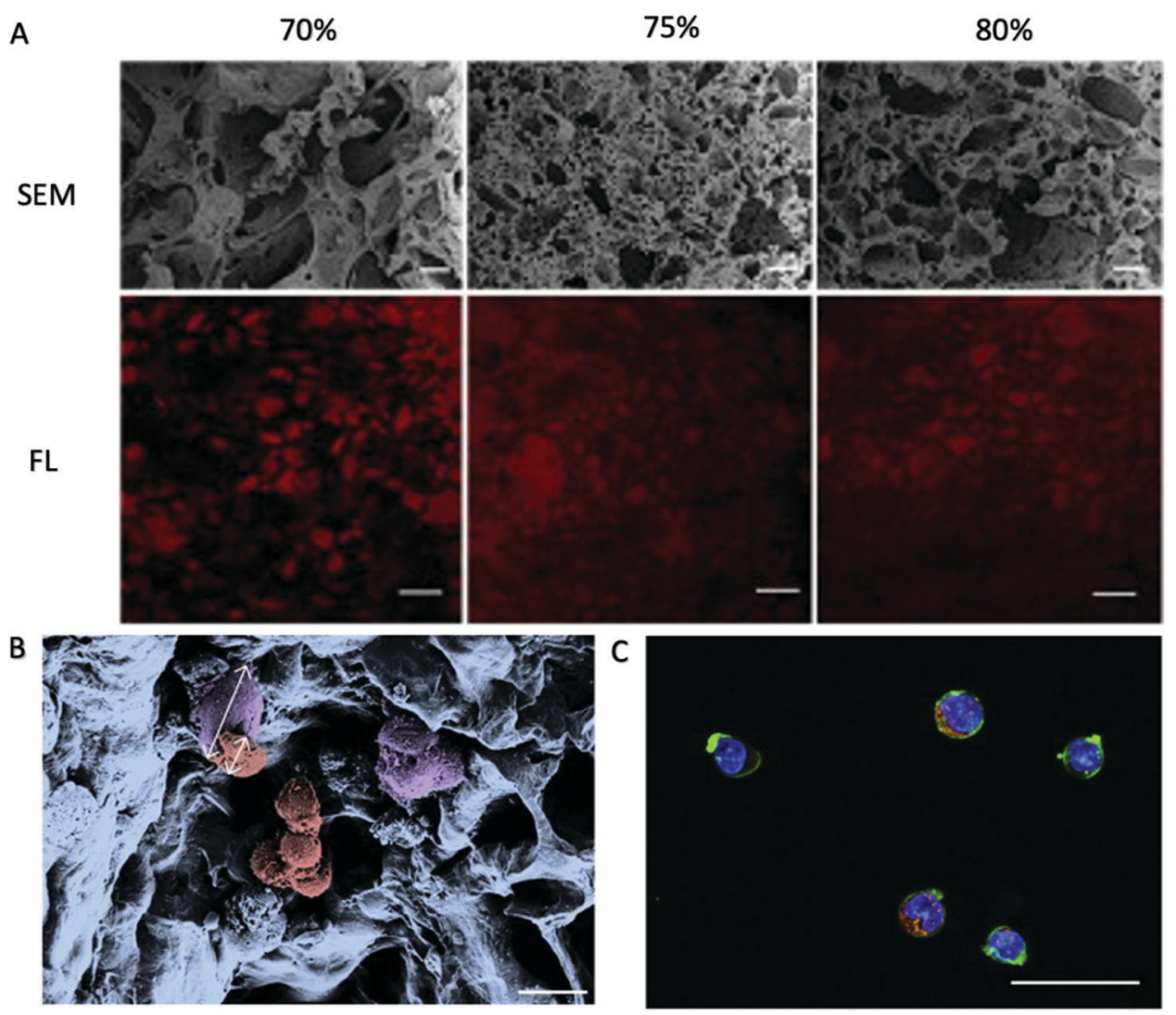

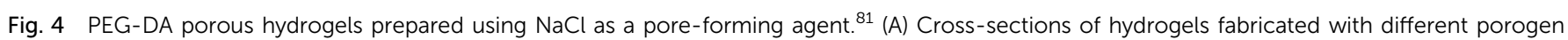

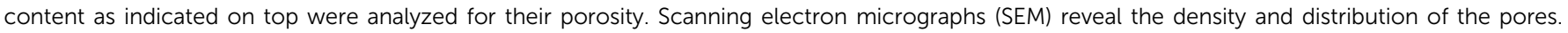

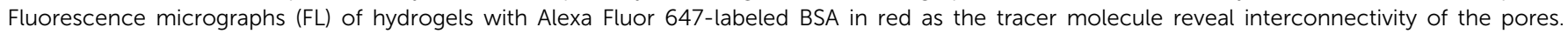

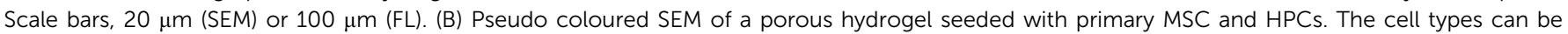

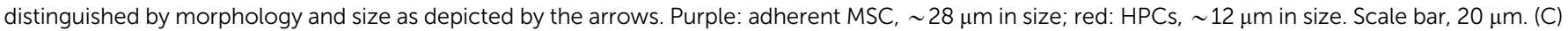

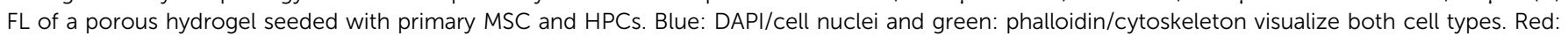
Alexa 568/CD34 identifies HPCs. Scale bar, $20 \mu \mathrm{m}$. Reproduced with Copyright Permission, 2014 Elsevier. 
unknown factors. Isern et al. used stromal stem cells, which constitute part of the same bone marrow niche as HSCs, to construct cell microspheres and found that the microspheres were the most effective in the long-term culture of HSCs (40-fold higher than the group without cell microspheres after four weeks). ${ }^{116}$ However, the mechanisms and secreted products within the cellular microspheres require further investigation (Fig. 6).

3D matrix coating platforms are mainly achieved by decellularized scaffolds. Decellularized materials are constructed from allogeneic or xenogeneic tissues upon the removal of internal cells through physical or chemical methods and are nonimmunogenic or low-immunogenic. Decellularized scaffolds are naturally complex in internal interconnection structures, and are difficult to isolate simply using porogen, salting or freeze-drying methods. The decellularized scaffold originated from a natural biological source, supporting its use as a platform for whole-organ reconstruction. Therefore, decellularized materials are advantageous in simulating the HSC niche. The most common physical method of lysing cells is rapid and repeated freeze-thawing, which generates ice crystals in the intracellular fluid, resulting in the rupture of the cell wall. Chemical reagents or enzymes are then used to remove the remaining cell contents. ${ }^{117}$ Alternatively, rapid changes in pressure can be used to lyse cells while washing with detergent to completely remove the cellular components. ${ }^{118}$

Bianco et al. decellularized bovine bone marrow and used it as an in vitro culture platform for HSCs and found that the decellularized scaffold was able to support the adhesion and expansion of HSCs while maintaining the phenotype of HSCs in vitro for a long time with the help of the cytokines secreted by stromal cells. Surprisingly, the transplanted HSCs preferentially adhered to areas corresponding to connective tissue surrounding the vascular region, recreating the natural vascular niche of HSCs in vitro. ${ }^{117}$ Nakamura et al. used HHP and sodium dodecyl sulfate
(SDS) detergent to decellularize cancellous bones. After being implanted into mice, the scaffold was found to be recellularized, stimulating the continuous homing of HSCs and promoting ectopic haematopoiesis. ${ }^{119}$ However, the use of SDS can cause damage to the vascular structure; therefore, decellularized scaffolds prepared by enzymatic digestion are recommended. In addition to bone marrow sources, decellularized scaffolds can be prepared on the basis of the ECM. Decellularized ECM scaffolds can promote the adhesion of HSCs and induce CD $34^{+}$cells to proliferate more than fourfold within seven days. ${ }^{120}$ Soffer-Tsur et al. used freezing and enzymatic digestion to decellularize the ECM and construct porous hydrogels for culturing HSCs. ${ }^{121} \mathrm{Li}$ et al. used a decellularized Wharton jelly matrix to construct a porous hydrogel scaffold and similarly demonstrated its unique advantage as a decellularized scaffold in promoting the maintenance of the phenotype of HSCs, enhancing cell self-renewal and maintaining the differentiation potential. ${ }^{122}$

The hypoxic niche is not closely integrated with other engineering strategies. ${ }^{86,123}$ Stem cell research requires a holistic view of HSC regulation, which requires a multi-parameter approach, and also aids in the development of new techniques to analyse the conditions of the HSC niche in vivo.

\section{Microscale technologies}

\subsection{Microspheres}

Microspheres are superior to other particle geometries for biomedical applications as they have a consistent shape and physical properties that ensure delivery stability and a large surface area that increases the efficiency of the coating and reduces the risk of immunization. ${ }^{125}$ Microspheres can be used as drug or cytokine carriers for therapeutic or tissue regenerative purposes. ${ }^{126}$ Liu et al. used poly(lactic-co-glycolic acid) (PLGA) microspheres as carriers of cytokines that are related to the
A

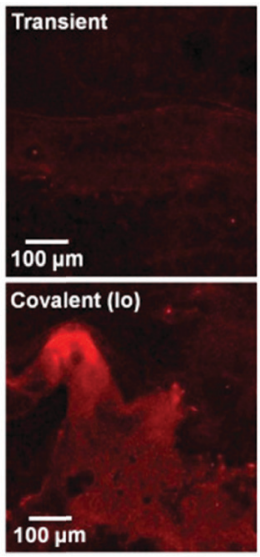

B

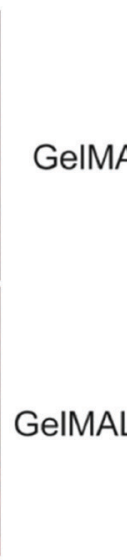

CellROX

Nucleus

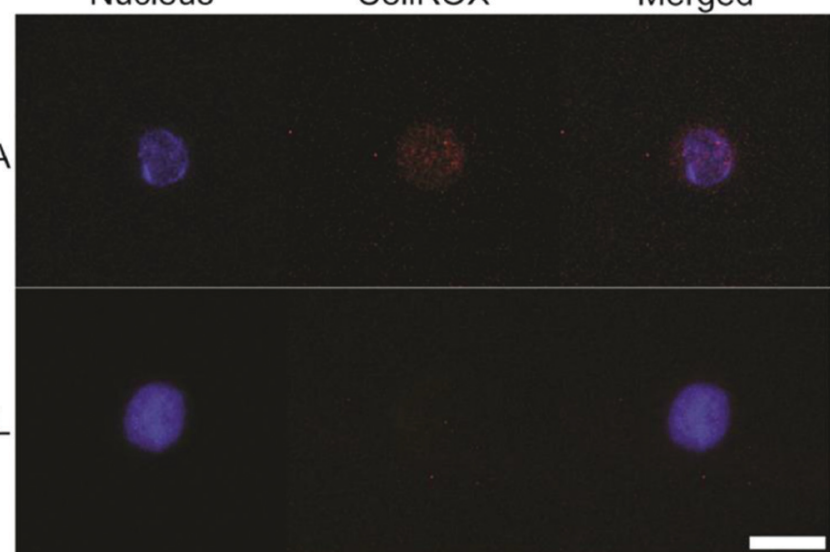

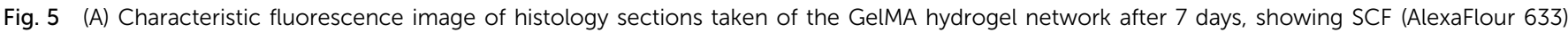

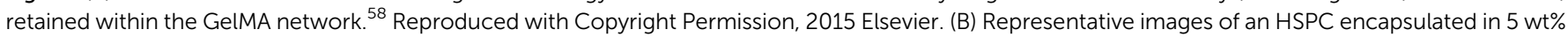

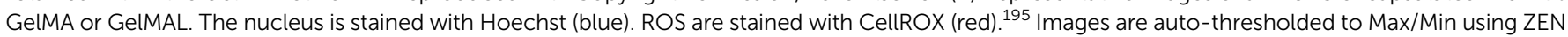
lite (Zeiss Microscopy). Scale bar is $10 \mu \mathrm{m}$. GelMAL = maleimide-functionalized gelatin. Reproduced with Copyright Permission, 2021 Elsevier. 

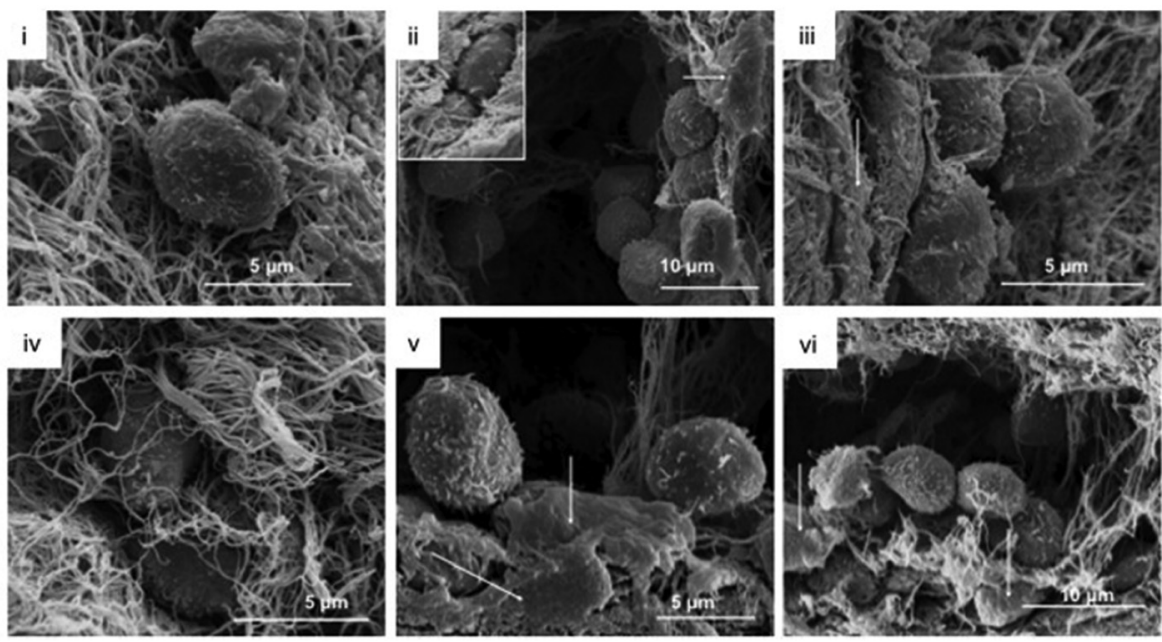

Fig. 6 Collagen gel topography and HPC morphology in the collagen gels were analyzed via SEM at day $14 .{ }^{198}$ MSC were located in direct association to HPC (arrows, ii, iii, v, vi). Reproduced with Copyright Permission, 2012 Elsevier.

regulation of HSCs to enhance angiogenesis in damaged muscle. ${ }^{127}$ In addition to be directly involved in the construction of the system, microspheres can be used for auxiliary characterization or be used as a crosslinker and reinforcer in the hydrogel. For example, Mahadik et al. used microfluids to simulate the bone marrow microenvironment in vitro, and later used fluorescent microspheres to construct gradient hydrogels, to help with the characterisation of hydrogel structures. ${ }^{128}$ Wang et al. used methacrylamide microspheres as a cross-linker and reinforcer of hydrogels. ${ }^{129}$

Microspheres can be made as scaffolds for culturing cells, mimicking the in vivo environment and functioning to maintain cell phenotypes or increase the proliferation rate of cells. ${ }^{130}$ Bello-Rodriguez et al. cultured MSCs onto collagen microspheres, covered the MSC encapsulated microspheres with a plasma coat, and then used this system to induce ectopic haematopoiesis in mice. ${ }^{131}$ Colloidal crystal microspheres were used as templates and later wrapped with sodium silicate or acrylamide hydrogels to form a HSC in vitro culture scaffold with an inverted colloidal crystal structure. The microsphere scaffolds were coated with a layer of nanomaterial to improve cell adhesion and were proved to support the proliferation and differentiation of $\mathrm{CD} 34^{+}$cells into B cells and to be essential for the production of $\mathrm{B}$ cells and antigen-specific antibodies. ${ }^{132}$ Fathi et al. used alginate and gelatine to encapsulate HSCs into microspheres, after which they examined changes in the state of cell differentiation towards NK cells and found that the microsphere scaffold affected the telomere length in the cells. ${ }^{133}$ Microspheres made using thermo-sacrificing materials such as gelatine can play the role of a porogen, as mentioned in Section 4.2, while microspheres made using materials that remain stable in the culture environment are used to construct micron-scale scaffolds and to verify the effect of microregulation on the fate of HSCs.

Typical methods used to produce microspheres include emulsion-solvent evaporation, sol-spray drying and electrospinning processes. ${ }^{126}$ The oil/water emulsion method involves the use of agitators to float the material as microdroplets in another phase of liquid, after which impurities are removed by washing or evaporation. ${ }^{125}$ The sol-spray drying is based on dispersing the material into tiny droplets of mist through an atomizer and removing the water in a stream of hot air to obtain a microsphere product. ${ }^{134}$ The electrospinning involves electrostatic charges on the surface of polymeric fluids under a high voltage electric field to generate microdroplets. ${ }^{135}$ However, electrospinning is more commonly used as a method of manufacturing nanofibres, which is explained in detail below.

\subsection{Microporous structure}

The scope of scaffolding applications in medicine has begun to shift from nondegradable synthetic materials and tissue grafts to degradable porous materials and regenerative medical tissues. ${ }^{50}$ In cases of porous scaffold materials, the appropriate porosity determines the ability of a scaffold to induce tissue regeneration and functionalization. ${ }^{136}$ The porous structure inside the scaffold results in a uniform distribution of cells, provides space for intercellular contact and stimulates cell proliferation and differentiation (when adapted to different cells). In addition, a porous scaffold is superior to a nonporous scaffold in terms of better nutrient and gas exchange, which is particularly important for tissue regeneration. Porous hydrogels with their macromolecular cross-linked network structure and additional pore space are uniquely suited to mimic the complex network structure of the ECM and are preferred for engineered artificial bone marrow analogues. ${ }^{81}$

The pore size of porous scaffolds can directly influence the behaviour and viability of cells. $5 \mu \mathrm{m}$ is a suitable pore size for vascular regeneration, while $20 \mu \mathrm{m}$ is suitable for cell growth. Pore sizes suitable for bone regeneration range from 100 to $350 \mu \mathrm{m}$, while tissue regeneration models require a pore size larger than $500 \mu \mathrm{m} .{ }^{50}$ The interconnected space inside a porous scaffold directs cell growth inwards, a process known as spontaneous phase transfer cell culture (PTCC) ${ }^{137}$ In cell-laden hydrogel constructs, the creation of microcavities introduces 
microedges, which are gel-cavity interfaces, into the bulk cell-embedded hydrogel. As non-anchorage-dependent species, the embedded cells tend to proliferate into isogenic groups (such as colonies) in non-cell-adhesive bulk gel. Cells residing in the gel-cavity interface were loaded with asymmetric encapsulating stress from different directions, with the stress from the gel bulk side being significantly greater than that from the cavity side. The net force of these unbalanced stresses guides and drives the proliferative expansion of these cells across boundaries from the gel phase towards the cavity phase, causing outgrowth from the bulk gel into the intra-gel cavities. These trans-phase outgrowing cells fill the open space inside the cavities upon further development. In addition to the mechanical impetus, the cell outgrowth direction also aligns with the ascending gradient of medium diffusion and nutrient exposure from a relatively concentrated phase towards a diffuse and ultimately void phase (cavity) in the bulk gel.

Traditionally, the porous hydrogels are made by solvent casting/particle leaching. The porogen with controllable particle size and the polymer solution are homogeneously mixed and solidified. The porous structure is then formed by melting and leaching the porogen through external changes such as in temperature, $\mathrm{pH}$, and other conditions or by dissolving the porogen. Sodium chloride $(\mathrm{NaCl})$ is often used to construct porous scaffolds for culturing HSCs. ${ }^{81,138}$

Pan et al. used $\mathrm{NaCl}$ as a porogen and adopted direct solvent casting to construct porous polycaprolactone (PCL) scaffolds. ${ }^{139}$ However, this PCL material has poor hydrophilicity and biological activity and is reported to exhibit low porosity with a large pore size, which is unfavourable for cell adhesion. ${ }^{140}$ Therefore, the PCL scaffold is not as effective as the fibrin or collagen scaffolds which exhibit high porosity and low fibre stiffness for promoting the expansion of HSCs in vitro. However, this limitation can be improved by adding modified silica into the scaffold. Yang et al. used vinyltrimethoxysilane to modify silica particles and then melted the particles within PCL. The silica/PCL scaffolds with $800 \mathrm{~nm}$ particles showed better performance in the surface roughness, hydrophilicity, and mechanical strength than PCL scaffolds, while the $100 \mathrm{~nm}$ silica/PCL scaffolds showed significantly better stem cell adhesion. ${ }^{141}$

Porous structures can also be generated using the stacking nanofibres made by electrospinning technology, ${ }^{142}$ which enables controllable thickness, composition and porosity of the nanofibres. Porous scaffolds made from these types of nanofibres benefit from a large surface area, exhibit enhanced cell adhesion, and can further be functionalized by incorporating growth factors during the manufacturing process to better control the proliferation and differentiation of cells cultured in the scaffold. However, the electrospinning technology is limited in producing more complex 3D shapes. ${ }^{143}$

In many cases, polymeric materials are made into nanofibres to build porous hydrogels. ${ }^{144}$ Polyether sulfone (PES) nanofibers have been shown to support $\mathrm{CD} 34^{+} \mathrm{CD} 45^{+}$cell expansion while promoting the expression of CXCR4 and lymphocyte functionassociated antigen-4 (LFA-4) in HSCs. ${ }^{145,146}$ Sabaghi et al. found that aminated PES nanofibres had a greater effect on the differentiation of megakaryocyte progenitors than conventional cultures, which was more conducive to accelerating platelet recovery and improving the transplantation success rate. ${ }^{147}$ Chua et al. also used electrospinning technology to construct polyethylene terephthalate (PET) nanofibre networks, followed by grafting poly(acrylic acid) onto the surface of the networks by photopolymerization and finally modifying the scaffolds by ammonification. The scaffolds with ethylene and butene spacers were found to have the greatest effect on $\mathrm{CD} 34^{+} \mathrm{CD} 45^{+}$cell expansion (a 200- and 235-fold increase, respectively), while those with hexene spacers maintained the maximum purity of $\mathrm{CD} 34^{+} \mathrm{CD} 45^{+}$cells $(41.1 \%){ }^{148}$ PET has also been used as a scaffold to study bone regeneration. For example, bone marrow stem cells and PET scaffolds were combined to repair Achilles tendon defects, enhance collagen regeneration and facilitate tissue healing. ${ }^{149}$ However, it is worth noting that the fibronectin-conjugated PET scaffold had a more pronounced effect on the expansion of HSCs, cell adhesion and the differentiation potential of subsequent in vitro cultures of $\mathrm{CD} 34^{+}$cells than a collagen-PET scaffold. ${ }^{150}$

Lyophilization, also known as freeze-drying, creates pores by generating thermodynamic instability within the material and causing phase separation through the rapid cooling and sublimation of specific solvents using vacuum technology. ${ }^{151}$ Severn et al. constructed an in vitro differentiation niche for HSCs based on a polyurethane (PU) scaffold by freeze-drying and found that the PU scaffold increased cell differentiation to the reticulocyte stage and maintained $\mathrm{CD} 34^{+}$cells during 28 days of culture but caused a high cell death rate. ${ }^{151}$ However, polymerized high internal phase emulsions (polyHIPEs) can be prepared with pendant thiol (-SH), which enables easy attachment of the molecule, and is applicable to more kinds of cells than PU scaffolds. ${ }^{152}$ Bai et al. constructed a star-like poly(carboxybetaine)based porous hydrogel through lyophilization. ${ }^{153}$ Within 24 days of culture, HSCs achieved 73-fold expansion in this zwitterionic hydrogel, which is better than a PEG hydrogel. The mechanism by which the zwitterionic hydrogel promotes the proliferation of HSCs may involve stimulating HSC self-renewal by inhibiting aerobic metabolism and inhibiting DNA-damaging reactive oxygen species (ROS) production. ${ }^{153}$ This mechanism is similar to that of UM-171 disruption of the accumulation of ROS by regulating inflammatory signalling, thereby driving HSC selfrenewal. ${ }^{19}$

Lyophilization can also be applied to natural materials such as heparin-chitosan scaffolds. ${ }^{154}$ Compared to the $14 \%$ maintenance rate of a $2 \mathrm{D}$ monolayer, the $3 \mathrm{D}$ chitosan-heparin scaffold exhibited a $17 \%$ maintenance rate and a $22 \%$ maintenance rate in a $5 \% \mathrm{O}_{2}$ hypoxic environment. ${ }^{154}$ Chitosan is commonly used to encapsulate HSCs together with sodium alginate or collagen. ${ }^{133,155}$

In addition, pores can be created by generating gas inside supporting materials under high pressure or by foaming agents. $^{50}$ For instance, Keskar et al. used the gas forming method to construct a poly(ethylene glycol) diacrylate (PEGDA) porous scaffold using sodium bicarbonate as a foaming agent to simulate the bone marrow niche. ${ }^{156}$ 


\subsection{Microchannels}

The construction of microchannels in hydrogel scaffolds creates a porous hydrogel derivative for various applications, and is used to further control and improve cell diffusion and transport in the hydrogel and enhance the mass transfer capacity of the scaffold. ${ }^{157}$ Miller et al. used carbohydrate glass as a biocompatible sacrificial material to construct homogeneous channels within the hydrogel. ${ }^{158}$ The creation of microchannels in a hydrogel can improve cell viability, promote cell spreading and direct cell extension. ${ }^{159,160}$ The most effective areas of material exchange in the culture environment and the highest rate of waste metabolism are found close to the transport channels, allowing for the maintenance of cell viability and function. The combination of this transport system and the porous structure of the scaffold makes the hydrogel more physically compatible with the human bone marrow cavity environment and facilitates the functional maintenance of HSCs.

A hydrogel-independent microchannel system is also capable of serving as a bioreactor for the in vitro culture of HSCs. Kresnowati et al. used microchannels to optimize the HSC cultures, avoiding tedious experimental procedures such as regular culture medium replenishment and possible contamination. In this system, a uniform distribution of nutrients is maintained, which is beneficial for HSC viability due to the stabilized oxygen levels and the increased cellular yield of the bioreactor. ${ }^{161}$.

Soft lithography micromoulding is often used for the construction of microchannels inside hydrogels. For example, soft lithography micromoulding is used to construct PLGA scaffolds that mimic microvessels. ${ }^{162}$ The construction process involves the manufacture of a semicylindrical photoresist template using a thermal reflow technique, topography of the PLGA over the template and, finally, stitching of the PLGA to build a scaffold with an internal microchannel structure.

Reconstructing microstructures is essential for the in vitro culture of HSCs; therefore, microfabrication techniques for scaffold modification to optimize cell viability, morphology and function while incorporating activating factors to maximally mimic the characteristics of the HSC niche is worth investigating.

\subsection{Microfluidics}

Microfluidics involves the precise control of fluids at the microscale, enabling the integration of the culture, isolation, detection, and analysis of cells at the micron scale and is a powerful tool for controlling the cellular environment. ${ }^{128,163}$ HSCs are nonadherent cells, making them difficult to be monitored individually for long periods of time using conventional cell culture methods. Instead, Faley et al. used a microfluidic platform to implement a $\mathrm{CD} 4^{+}$single-cell isolation assay system using passive capture for microscale drug response detection. ${ }^{164}$ Han et al. similarly achieved the capture of individual HSCs with the help of microfluidic devices, enabling high-throughput analysis of the motility and behaviour of individual cells. ${ }^{165}$

In addition to the fluid state of the internal microenvironment of bone marrow, the cellular diversity and complex functions of living bone marrow cannot be simulated using traditional plate cultures or scaffolds. Therefore, to test the method of culturing live bone marrow in vitro, Torisawa et al. induced new bone formation in vivo in mice, removed the engineered bone after the eighth week and transplanted it to an in vitro culture platform based on microfluidic technology. Live bone marrow on the microfluidic platform independently produced cytokines that maintain haematopoietic function in vitro and help restore haematopoietic niche function. At the same time, the bone marrow in vitro maintains HSCs in their spatial location in the original niche, allowing HSCs to remain at their normal ratio. ${ }^{166}$

Microfluidic devices can also serve as new tools for the longterm detection of stem cell behaviour in vitro and for the study of stem cell niches, providing a new direction to break the current HSCT dilemma with respect to stem cell shortages. Sieber et al. used a zirconia scaffold to mimic the porous and rigid nature of cancellous bone microstructures, created a microenvironment similar to the bone marrow niche using microfluidics and found that $31.71 \%$ of the cells maintained a CD $34^{+} \mathrm{CD} 38^{-}$phenotype after 28 days. ${ }^{167}$ Carreras et al. used microfluidics to construct bilayer microdroplets with MSCs on the inside and HSCs on the outside, and based on this coculture model, they achieved sustainable expansion of CD34 ${ }^{+}$cells in vitro for eight weeks. ${ }^{111}$

\subsection{Bioprinting}

The internal space and topology of scaffolds cannot be precisely designed for either by porogen or by electrospinning technology. ${ }^{168}$ Photolithography requires moulds for fabricating internal microchannels, which is costly and cannot be built directly into the final scaffold. ${ }^{169}$ However, the use of bioprinting in constructing the HSC artificial niche can circumvent these problems and efficiently create a flexible architecture of the internal space of the scaffold at a low cost. ${ }^{168,170}$ In addition, bioprinting can easily modify the scaffold structure at the microscopic level, with a high degree of flexibility and consistency of the constructed scaffolds, which is essential for the tissue engineering of the HSC niche. ${ }^{169}$

There are three categories of bioprinting systems: bioplotting, inkjet printing and light-based printing. ${ }^{169}$ Bioplotting, also known as extrusion printing, is based on extrusion equipment printing the hydrogel fibers, which are then deposited layer by layer to form a 3D structure. ${ }^{171}$ This approach was used by Pati et al. to construct scaffolds suitable for long-term cell culture with PCL and the ECM. ${ }^{172}$ Inkjet printing involves spraying material in the form of droplets, building 2D layers of material, and subsequently building scaffolds with complex internal structures in a superimposed manner. ${ }^{173}$ Due to the advantages of inkjet printing in constructing 2D structures, this method is used to construct micropatterns on polymers and later control the stem cell adhesion geometry. ${ }^{174}$

There are two forms of light-based bioprinting: digital light processing (DLP) and laser-assisted printing. ${ }^{170}$ DLP is a fulllayer printing process in which the entire plane of an optical pattern is projected onto a photosensitive polymer solution, significantly reducing the manufacturing time and eliminating 
connecting interfaces between materials. ${ }^{175,176}$ The hydrogel material used in this method is mainly methacrylamidefunctionalized gelatine (GelMA). ${ }^{177}$ Laser-assisted printing enables direct shaping of the support material by laser beams without the use of a mask. This enables the construction of more complex scaffold structures in a single process step and can be applied from the submicron to the centimetre level. ${ }^{178}$ Table 3 lists and compares four different types of bioprinting.

\section{Construction and dissolution of hydrogel scaffolds}

\subsection{Hydrogels for haematopoietic stem cell culture}

The 3D porous structure of a hydrogel has a suitable spatial structure and porosity, which is conducive to the adhesion, growth, and proliferation of stem cells. In addition, hydrogels have good biocompatibility and can be stably combined with stem cells. A high surface activity is conducive to the combination of the stem cells and scaffold, and promotes the proliferation and phenotype maintenance of HSCs. ${ }^{180}$ The hydrogels show a similar mechanical strength to the ECM, and are easy to mould, which stabilize the growth space for HSCs. Implantable hydrogels are biodegradable and can self-degrade in the body. The degradation rate needs to be adjusted within an appropriate range to ensure the growth of other tissues is not affected while the growth of HSCs is supported. ${ }^{181}$ Research on in vitro HSC cultures with a hydrogel focuses on the expansion of the HSCs. A variety of materials have been proved to significantly promote the expansion of HSCs. ${ }^{3,10,100}$ However, whether the cells inside the hydrogel are accessible or not needs to be taken into consideration. This is because the main objective of in vitro expansion studies of HSC is to obtain transplantable stem cells. Therefore, materials that can be degraded with minimal damage to cells have been valued to minimize unavailable HSCs which are useless for HSCT. A typical example is the sodium alginate hydrogel that can be removed by sodium citrate. And to address the problem of lysis of polymeric hydrogels, researchers used the addition of peptides to the polymeric material to facilitate the removal of cells from the hydrogel by enzymatic dissolution at the end of the culture. ${ }^{153}$ This introduction of peptide self-assembly properties into polymers, thereby imparting biological properties to polymer hydrogels, such as enzymatic degradation, can effectively eliminate the limitations imposed by the material itself (Table 4). ${ }^{182}$

\subsection{Using ion exchange reactions to construct hydrogels}

The ion exchange reaction is a process in which the cations or anions in the exchange agent are reversibly exchanged with ions of the same kind in the material solution. ${ }^{183}$ Through the ion exchange reaction, the functional units of the material build up and form a cross-linked network structure, resulting in a hydrogel. ${ }^{184}$

Alginate is a polysaccharide isolated from seaweed with mild gelling properties in the presence of divalent ions (e.g., calcium). ${ }^{185}$ The limited range of the mechanical properties of alginate limits its application, but meets the requirements for use as a scaffold for HSC in vitro proliferation. ${ }^{186}$ Alginate can be de-cross-linked, allowing the cells inside the scaffold to be collected for further analysis at the end of the culture period. Yuan et al. cultured HSCs with sodium alginate, and the percentage of $\mathrm{CD}^{+} 4^{+}$cells increased from $2.60 \pm 0.52 \%$ to $13.27 \pm 2.65 \%$ after only 12 days of culture. ${ }^{187}$ To maintain the porosity and permeability of the deposited structure of an alginate system while increasing the Young's modulus of the scaffold, Moore et al. used methylcellulose to modify sodium alginate and produce a bioink suitable for 3D printing. The combination of $6 \%$ methylcellulose and $2 \%$ alginate was found to best mimic the optional properties of bone marrow properties, such as the overall structure, oxygen availability and rheological properties. ${ }^{188}$ Braham et al. then added an additional external mechanical stimulus, intermittent hydrostatic pressure (IHP), based on sodium alginate to simulate the mechanical environment of osteoblasts within the bone marrow. The combination of osteoblasts and IHP resulted in an increase in the migration rate of HSCs while maintaining a high survival rate. ${ }^{189}$

\subsection{Using a photochemical reaction to construct hydrogels}

Photoinitiators can be initiated by ultraviolet or visible light, participate in the chain polymerization reaction of monomers, and quickly form a highly cross-linked polymer network. ${ }^{190} \mathrm{~A}$ common HSC in vitro culture scaffold constructed via photochemical reaction is the methacrylamide-functionalized hydrogel.

Gilchrist et al. used lithium acylphosphinate as a photoinitiator to construct GelMA hydrogels and used them as an exogenous stimulus to increase the number of haematopoietic

Table 3 The pros and cons of bioprinting $169,170,175,179$

\begin{tabular}{|c|c|c|c|}
\hline Type & Principle and resolution & Advantages & Disadvantages \\
\hline Bioplotting & $\begin{array}{l}\text { Extrusion equipment presses out } \\
\text { the scaffold material in a line } \\
(50 \mu \mathrm{m})\end{array}$ & Low cost and easy to operate & $\begin{array}{l}\text { Causes damage to the cells, the process is slow, the nozzle } \\
\text { easily clogs, and the structure of the built-up scaffold is } \\
\text { simple }\end{array}$ \\
\hline $\begin{array}{l}\text { Inkjet } \\
\text { printing }\end{array}$ & $\begin{array}{l}\text { The scaffold material is ejected } \\
\text { in the form of liquid droplets } \\
(50 \mu \mathrm{m})\end{array}$ & $\begin{array}{l}\text { Moderate build speed, low damage to } \\
\text { cells, low equipment cost and simple } \\
\text { operation. }\end{array}$ & $\begin{array}{l}\text { Simple structure of the built-up scaffold, and the nozzle is } \\
\text { easily clogged }\end{array}$ \\
\hline DLP & $\begin{array}{l}\text { Full-width printing through } \\
\text { masks }(1 \mu \mathrm{m})\end{array}$ & High speed & $\begin{array}{l}\text { High cost, complex operation, most of the initiators are } \\
\text { toxic, and require additional mask design }\end{array}$ \\
\hline $\begin{array}{l}\text { Laser } \\
\text { assisted }\end{array}$ & $\begin{array}{l}\text { A direct laser-writing technology } \\
(<500 \mathrm{~nm})\end{array}$ & Supports submicron scale scaffolds & High equipment costs and high waste of raw materials \\
\hline
\end{tabular}


Table 4 The pros and cons of three methods in constructing hydrogels ${ }^{10,51,185,198}$

Type Advantages

Ion exchange reaction Time efficient and low cellular damage

Photochemical reaction Ability to build complex structures

Inability to build complex inner structures Self-assembling Low cellular damage and superiority in mimicking cell growth environments Long gel formation time

progenitors and increased the progeny by $7.52 \pm 3.65$-fold over a seven-day culture period. ${ }^{191}$ Interestingly, Gilchrist et al. found that MSC-mediated matrix remodelling may contribute to a dynamic culture environment and promote retention of quiescent HSC populations when GelMA is used as a coculture scaffold for MSCs and HSCs. ${ }^{192}$ This outcome may have been due to MSC density-dependent upregulation and the altered mechanical properties of the hydrogel. For the maintenance of quiescence, Mahadik et al. found that in the GelMA hydrogel system, covalent SCF was effective in maintaining the phenotype of HSCs and preserving the quiescent state, while soluble SCF significantly enhanced the proliferation and differentiation of HSCs. ${ }^{58}$ This finding has implications for HSC amplification and genealogical specification.

Zhang et al. then used lithium phenyl-2,4,6-trimethylbenzoylphosphinate as a photoinitiator and methacrylic anhydride (MA) to functionalize hyaluronic acid (HA) to construct HAMA hydrogels. ${ }^{193}$ They enhanced the physical properties of the hydrogel using carbon nanotubes while supporting the scavenging ability of the culture system for oxides and peroxides. In addition, the proliferation ability and pluripotency of HSCs were improved by inhibition of the ROS pathway. HAMA and carbon nanotube scaffolds provide a new strategy for establishing artificial niches for HSCs with low ROS levels. At the end of the cell culture, the GelMA lysis solution can be used to lyse the hydrogel at the end of the culture to facilitate the removal of the cells, but the lysis time is not advantageous compared to sodium alginate hydrogels (Fig. 5). ${ }^{194}$

\subsection{Self-assembling hydrogels}

Some materials can self-assemble and form ordered structures without external intervention, which can then be applied to simulate ECM structures in the field of regenerative medicine. ${ }^{196,197}$ Self-assembled hydrogels can be hydrolyzed enzymatically, reducing the damage to cells during hydrogel removal.

The main natural self-assembling materials applied for the in vitro expansion of HSC are collagen, fibrin and matrigel. Collagen is the main component of the ECM and is superior to synthetic materials in facilitating the amplification of HSCs. ${ }^{140}$ It is reported that HSCs were able to migrate in collagen fibres assisted by spindle MSCs. And interestingly, bone marrowderived MSCs enhanced the maintenance of the cellular CD $34^{+} \mathrm{CD} 38^{-}$phenotype in the collagen scaffold, whereas cord blood-derived MSCs promoted the proliferation and differentiation of HSCs. ${ }^{198}$ Collagen can also be used as a scaffold material together with synthetic materials. For example, collagen and PCL were combined to construct an artificial niche, achieving 20 -fold proliferation of $\mathrm{CD}^{+} 4^{+}$cells within ten days of culture. ${ }^{199}$
The fibrin network in the body mainly protects neutrophils, fibroblast infiltration and macrophages while combining collagen, fibronectin, elastin and laminin to form a repair structure. ${ }^{200}$ Fibrin is superior to other extracellular matrices in mediating signalling between cells. ${ }^{201}$ This superiority is reflected in the maintenance and expansion support of HSCs by fibrin scaffolds. ${ }^{140}$ Garcia-Abrego et al. used fibrin to construct an artificial niche simulating foetal liver and found that a soft fibrin hydrogel (0.6 $\mathrm{mg} \mathrm{mL}^{-1}$ fibrinogen) with an elastic modulus value of $0.78 \pm 0.10 \mathrm{kPa}$ best mimics the foetal liver in terms of hardness of the liver niche and allowed the proliferation of HSCs and differentiated progeny cells. ${ }^{202}$ In contrast, the fibronectin in fibrinogenesis was used as a coating material to modify PES or poly-L-lactic acid. ${ }^{203,204}$ Notably, the results showed that fibronectin-coated scaffolds facilitated the expansion of $\mathrm{CD} 34^{+}$cells.

Matrigel is extracted from the ECM of protein-rich mouse tumours and has a complex composition that can be undesirable when studying specific signalling pathways but can be used as a scaffolding material to support the expansion of HSCs in vitro. ${ }^{189}$ However, the promotion of cell differentiation by these matrix gels is more pronounced, leading to the most frequent matrix gel applications in cell regeneration studies. ${ }^{205}$

For natural self-assembling materials, their physicochemical properties can hardly be modified to mimic specific cell growth environments. In contrast, synthetic self-assembling biomaterials can be designed and modified to mimic specific cellular microenvironments and key elements of ECM components. ${ }^{206}$ Raic et al. generated an ECM-like environment within the PEG-DA hydrogel to enhance the promotion of HSC expansion by MSCs in vitro by using RGD as an artificial ECM additive to increase the adhesion of PEG-based hydrogels with HSCs. ${ }^{81}$ The addition of RGD functional peptide also provided self-assembly capabilities to PEG-DA hydrogels. This combination of the functional properties or natural structures of self-assembled peptides with biomaterials gives the material a completely new bioactive function. ${ }^{207}$ In the future, a large number of molecular tools can be used to facilitate the pioneering design of synthetic biomaterials and to provide impetus for the development of clinical applications.

\section{Conclusions}

Various attempts for in vitro proliferation of HSCs using biochemical and physical strategies have been made to break through the barriers of existing stem cell science research and make progress in the regeneration of haematopoietic systems. HSC niche mimics engineered using biochemical bionics, biomimetic materials and microscale technologies, which is a multidisciplinary process, have been summarized 
and elaborated in this review. Efforts to engineer human organ tissues by combining the semiconductor industry, biology and materials science are being made. Currently, effective methods for the in vitro expansion of HSCs are coculture systems, or the use of decellularized scaffolds to construct an in vitro ECM environment. However, the clinical translation of these methods is still hindered by their low cost-efficiency. The current systems are still unable to meet the need for rapid and substantial amplification of the HSCs while maintaining phenotypes in short term culture, and efficient long-term culture of HSCs. Moreover, the study of regulatory factors and signalling cascades in the preparation of HSCs by inducing differentiation of pluripotent stem cells remains unclear. It is also a long way from clinical application for ethical reasons. In the future, with the in-depth combination of bioprinting and stem cell research, a breakthrough in the field of haematopoiesis is expected. Furthermore, with the improvement of bioinks, it is possible to simulate the biochemical and complex physical environment of an ECM simply utilizing hydrogels.

\section{Conflicts of interest}

There are no conflicts to declare.

\section{Acknowledgements}

This work was supported by grants from the City University of Hong Kong (SGP 9380099, 9231412, and 7005628 to Dong-An Wang), a grant from the Karolinska Institutet Ming Wai Lau Centre of Reparative Medicine (to Dong-An Wang), and by the Lvyangjinfeng Talent Program of Yangzhou (to Hang Yao).

\section{References}

1 S. J. Morrison, N. Uchida and I. L. Weissman, The biology of hematopoietic stem cells, Annu. Rev. Cell Dev. Biol., 1995, 11(1), 35-71.

2 C. J. Eaves, Hematopoietic stem cells: concepts, definitions, and the new reality, Blood, 2015, 125(17), 2605-2613.

3 A. C. Wilkinson, K. J. Igarashi and H. Nakauchi, Haematopoietic stem cell self-renewal in vivo and ex vivo, Nat. Rev. Genet., 2020, 21(9), 541-554.

4 S. H. Orkin and L. I. Zon, Hematopoiesis: an evolving paradigm for stem cell biology, Cell, 2008, 132(4), 631-644.

5 Q. Wei and P. S. Frenette, Niches for hematopoietic stem cells and their progeny, Immunity, 2018, 48(4), 632-648.

6 P. Zhang, et al., The physical microenvironment of hematopoietic stem cells and its emerging roles in engineering applications, Stem Cell Res. Ther., 2019, 10(1), 1-13.

7 L. Li and T. Xie, Stem cell niche: structure and function, Annu. Rev. Cell Dev. Biol., 2005, 21, 605-631.

8 L. I. Shlush, Age-related clonal hematopoiesis, Blood, 2018, 131(5), 496-504.
9 L. E. Portillo Esquivel and B. Zhang, Application of Cell, Tissue, and Biomaterial Delivery in Cardiac Regenerative Therapy, ACS Biomater. Sci. Eng., 2021, 7(3), 1000-1021.

10 A. Congrains, et al., 3D Scaffolds to Model the Hematopoietic Stem Cell Niche: Applications and Perspectives, Materials, 2021, 14(3), 569.

11 G. Ingavle, A. Vaidya and V. Kale, Constructing ThreeDimensional Microenvironments Using Engineered Biomaterials for Hematopoietic Stem Cell Expansion, Tissue Eng., Part B, 2019, 25(4), 312-329.

12 T. Terwilliger and M. Abdul-Hay, Acute lymphoblastic leukemia: a comprehensive review and 2017 update, Blood Cancer J., 2017, 7(6), e577.

13 N. Baryawno, et al., A cellular taxonomy of the bone marrow stroma in homeostasis and leukemia, Cell, 2019, 177(7), 1915.e16-1932.e16.

14 D. Duarte, E. D. Hawkins and C. Lo, Celso, The interplay of leukemia cells and the bone marrow microenvironment, Blood, 2018, 131(14), 1507-1511.

15 T. H. Ambrosi, et al., Adipocyte accumulation in the bone marrow during obesity and aging impairs stem cell-based hematopoietic and bone regeneration, Cell Stem Cell, 2017, 20(6), 771.e6-784.e6.

16 M. Maryanovich, et al., Adrenergic nerve degeneration in bone marrow drives aging of the hematopoietic stem cell niche, Nat. Med., 2018, 24(6), 782-791.

$17 \mathrm{~S}$. Valletta, et al., Micro-environmental sensing by bone marrow stroma identifies IL- 6 and TGF $\beta 1$ as regulators of hematopoietic ageing, Nat. Commun., 2020, 11(1), 1-13.

18 E. A. Copelan, Hematopoietic stem-cell transplantation, $N$. Engl. J. Med., 2006, 354(17), 1813-1826.

19 A. C. Wilkinson and H. Nakauchi, Stabilizing hematopoietic stem cells in vitro, Curr. Opin. Genet. Dev., 2020, 64, 1-5.

20 A. Gratwohl, et al., Hematopoietic stem cell transplantation: a global perspective, JAMA, 2010, 303(16), 1617-1624.

21 A. Ivanovs, et al., Identification of the niche and phenotype of the first human hematopoietic stem cells, Stem Cell Rep., 2014, 2(4), 449-456.

22 A. P. $\mathrm{Ng}$ and W. S. Alexander, Haematopoietic stem cells: past, present and future, Cell Death Discovery, 2017, 3(1), 1-4.

23 S. J. Morrison and D. T. Scadden, The bone marrow niche for haematopoietic stem cells, Nature, 2014, 505(7483), 327-334.

$24 \mathrm{~J}$. Zhang, et al., Identification of the haematopoietic stem cell niche and control of the niche size, Nature, 2003, 425(6960), 836-841.

25 F. Arai, et al., $\mathrm{Tie}_{2}$ /angiopoietin-1 signaling regulates hematopoietic stem cell quiescence in the bone marrow niche, Cell, 2004, 118(2), 149-161.

26 J. Lévesque, F. Helwani and I. Winkler, The endosteal 'osteoblastic'niche and its role in hematopoietic stem cell homing and mobilization, Leukemia, 2010, 24(12), 1979-1992.

27 T. T. Lau and D.-A. Wang, Stromal cell-derived factor-1 (SDF-1): homing factor for engineered regenerative medicine, Expert Opin. Biol. Ther., 2011, 11(2), 189-197. 
28 M. J. Kiel, et al., SLAM family receptors distinguish hematopoietic stem and progenitor cells and reveal endothelial niches for stem cells, Cell, 2005, 121(7), 1109-1121.

29 P. Ramalingam, J. M. Butler and M. G. Poulos, Vascular Regulation of Hematopoietic Stem Cell Homeostasis, Regeneration, and Aging. Current, Stem Cell Rep., 2021, 1-10.

30 L. Ding, et al., Endothelial and perivascular cells maintain haematopoietic stem cells, Nature, 2012, 481(7382), 457-462.

31 A. C. Gomes, M. Saraiva and M. S. Gomes, The bone marrow hematopoietic niche and its adaptation to infection, Seminars in Cell \& Developmental Biology, Elsevier, 2020.

32 T. Sugiyama, et al., Maintenance of the hematopoietic stem cell pool by CXCL12-CXCR4 chemokine signaling in bone marrow stromal cell niches, Immunity, 2006, 25(6), 977-988.

33 A. Mendelson and P. S. Frenette, Hematopoietic stem cell niche maintenance during homeostasis and regeneration, Nat. Med., 2014, 20(8), 833-846.

34 S. Kaur, et al., Self-repopulating recipient bone marrow resident macrophages promote long-term hematopoietic stem cell engraftment, Blood, 2018, 132(7), 735-749.

35 S. Yamazaki, et al., Nonmyelinating Schwann cells maintain hematopoietic stem cell hibernation in the bone marrow niche, Cell, 2011, 147(5), 1146-1158.

36 T. Hoshiba, et al., Decellularized extracellular matrix as an in vitro model to study the comprehensive roles of the ECM in stem cell differentiation, Stem Cells Int., 2016, 6397820.

37 A. Pardo-Saganta, et al., Role of the extracellular matrix in stem cell maintenance, Curr. Stem Cell Rep., 2019, 5(1), 1-10.

38 A. Nakamura-Ishizu, et al., Extracellular matrix protein tenascin-C is required in the bone marrow microenvironment primed for hematopoietic regeneration, Blood, 2012, 119(23), 5429-5437.

39 H. Yoshihara, et al., Thrombopoietin/MPL signaling regulates hematopoietic stem cell quiescence and interaction with the osteoblastic niche, Cell Stem Cell, 2007, 1(6), 685-697.

$40 \mathrm{H}$. Qian, et al., Contribution of $\alpha 6$ integrins to hematopoietic stem and progenitor cell homing to bone marrow and collaboration with $\alpha 4$ integrins, Blood, 2006, 107(9), 3503-3510.

41 A. J. Potocnik, C. Brakebusch and R. Fässler, Fetal and adult hematopoietic stem cells require $\beta 1$ integrin function for colonizing fetal liver, spleen, and bone marrow, Immunity, 2000, 12(6), 653-663.

42 T. Mammoto and D. E. Ingber, Mechanical control of tissue and organ development, Development, 2010, 137(9), 1407-1420.

43 J. A. Spencer, et al., Direct measurement of local oxygen concentration in the bone marrow of live animals, Nature, 2014, 508(7495), 269-273.
44 U. Blank and S. Karlsson, TGF- $\beta$ signaling in the control of hematopoietic stem cells, Blood, 2015, 125(23), 3542-3550.

45 M. Decker, et al., Hepatic thrombopoietin is required for bone marrow hematopoietic stem cell maintenance, Science, 2018, 360(6384), 106-110.

$46 \mathrm{X}$. Huang, et al., Co-cultured h BMSC s and HUVEC s on human bio-derived bone scaffolds provide support for the long-term ex vivo culture of HSC/HPC s, J. Biomed. Mater. Res., Part A, 2016, 104(5), 1221-1230.

47 Y. C. Shin, et al., RGD peptide and graphene oxide cofunctionalized PLGA nanofiber scaffolds for vascular tissue engineering, Regener. Biomater., 2017, 4(3), 159-166.

$48 \mathrm{H}$. Kobayashi, et al., Environmental optimization enables maintenance of quiescent hematopoietic stem cells ex vivo, Cell Rep., 2019, 28(1), 145.e9-158.e9.

49 C. Gerri, et al., Hif- $1 \alpha$ and Hif- $2 \alpha$ regulate hemogenic endothelium and hematopoietic stem cell formation in zebrafish, Blood, 2018, 131(9), 963-973.

50 S. J. Hollister, Porous scaffold design for tissue engineering, Nat. Mater., 2005, 4(7), 518-524.

51 M. P. Lutolf and H. M. Blau, Artificial stem cell niches, $A d v$. Mater., 2009, 21(32-33), 3255-3268.

52 L. G. Schuettpelz, et al., G-CSF regulates hematopoietic stem cell activity, in part, through activation of Toll-like receptor signaling, Leukemia, 2014, 28(9), 1851-1860.

53 C. A. Oedekoven, et al., Hematopoietic stem cells retain functional potential and molecular identity in hibernation cultures, Stem Cell Rep., 2021, 16(6), 1614-1628.

54 A. Bernad, et al., Interleukin-6 is required in vivo for the regulation of stem cells and committed progenitors of the hematopoietic system, Immunity, 1994, 1(9), 725-731.

55 F. Oubari, et al., The important role of FLT3-L in ex vivo expansion of hematopoietic stem cells following co-culture with mesenchymal stem cells, Cell J., 2015, 17(2), 201.

56 J. S. Choi, B. P. Mahadik and B. A. Harley, Engineering the hematopoietic stem cell niche: Frontiers in biomaterial science, Biotechnol. J., 2015, 10(10), 1529-1545.

57 V. Barnhouse, et al., Perivascular secretome influences hematopoietic stem cell maintenance in a gelatin hydrogel, Ann. Biomed. Eng., 2021, 49(2), 780-792.

58 B. P. Mahadik, et al., The use of covalently immobilized stem cell factor to selectively affect hematopoietic stem cell activity within a gelatin hydrogel, Biomaterials, 2015, 67, 297-307.

$59 \mathrm{~J}$. Friel, et al., Diverse isoforms of colony-stimulating factor-1 have different effects on the development of stroma-dependent hematopoietic cells, J. Cell. Physiol., 2005, 204(1), 247-259.

60 M. Yamashita and E. Passegué, TNF- $\alpha$ coordinates hematopoietic stem cell survival and myeloid regeneration, Cell Stem Cell, 2019, 25(3), 357.e7-372.e7.

61 M. Etzrodt, et al., Inflammatory signals directly instruct PU. 1 in HSCs via TNF, Blood, 2019, 133(8), 816-819.

62 S. Kumar and H. Geiger, HSC niche biology and HSC expansion ex vivo, Trends Mol. Med., 2017, 23(9), 799-819.

63 S. S. Akhkand, et al., Evaluation of umbilical cord blood CD34+ hematopoietic stem cells expansion with inhibition 
of TGF- $\beta$ receptorII in co-culture with bone marrow mesenchymal stromal cells, Tissue Cell, 2016, 48(4), 305-311.

$64 \mathrm{~J}$. Li, et al., In Vitro Expansion of Hematopoietic Stem Cells by Inhibition of Both GSK3 and p38 Signaling, Stem Cells Dev., 2019, 28(22), 1486-1497.

65 K. Sudo, et al., Polyvinyl alcohol hydrolysis rate and molecular weight influence human and murine HSC activity ex vivo, Stem Cell Res., 2021, 56, 102531.

66 A. C. Wilkinson, et al., Long-term ex vivo haematopoieticstem-cell expansion allows nonconditioned transplantation, Nature, 2019, 571(7763), 117-121.

67 D. A. Breems, E. Blokland and R. Ploemacher, Stromaconditioned media improve expansion of human primitive hematopoietic stem cells and progenitor cells, Leukemia, 1997, 11(1), 142-150.

68 Y. Zhang, et al., Human placenta-derived mesenchymal progenitor cells support culture expansion of long-term culture-initiating cells from cord blood CD34+ cells, Exp. Hematol., 2004, 32(7), 657-664.

69 J.-J. Lataillade, et al., Stromal cell-derived factor 1 regulates primitive hematopoiesis by suppressing apoptosis and by promoting G0/G1 transition in CD34+ cells: evidence for an autocrine/paracrine mechanism, Blood, 2002, 99(4), 1117-1129.

70 M. Ajami, et al., Comparison of cord blood CD34+ stem cell expansion in coculture with mesenchymal stem cells overexpressing SDF-1 and soluble/membrane isoforms of SCF, J. Cell. Biochem., 2019, 120(9), 15297-15309.

71 I.-I. Kuan, et al., EpEX/EpCAM and Oct4 or Klf4 alone are sufficient to generate induced pluripotent stem cells through STAT3 and HIF2 $\alpha$, Sci. Rep., 2017, 7(1), 1-14.

72 Q. Li, et al., Wharton's jelly mesenchymal stem cell-based or umbilical vein endothelial cell-based serum-free coculture with cytokines supports the ex vivo expansion/maintenance of cord blood hematopoietic stem/progenitor cells, Stem Cell Res. Ther., 2019, 10(1), 1-9.

73 Y.-M. Kook, et al., Design of biomimetic cellular scaffolds for co-culture system and their application, J. Tissue Eng., 2017, 8, 1-17, DOI: 2041731417724640.

74 D. Lachowski, et al., Matrix stiffness modulates the activity of MMP-9 and TIMP-1 in hepatic stellate cells to perpetuate fibrosis, Sci. Rep., 2019, 9(1), 1-9.

75 D. Jhala and R. Vasita, A review on extracellular matrix mimicking strategies for an artificial stem cell niche, Polym. Rev., 2015, 55(4), 561-595.

$76 \mathrm{~J}$. Holst, et al., Substrate elasticity provides mechanical signals for the expansion of hemopoietic stem and progenitor cells, Nat. Biotechnol., 2010, 28(10), 1123-1128.

77 J. S. Choi and B. A. Harley, Marrow-inspired matrix cues rapidly affect early fate decisions of hematopoietic stem and progenitor cells, Sci. Adv., 2017, 3(1), e1600455.

78 L. Rödling, et al., 3D models of the hematopoietic stem cell niche under steady-state and active conditions, Sci. Rep., 2017, 7(1), 1-15.

79 Y. Dong, et al., Nanotechnology shaping stem cell therapy: Recent advances, application, challenges, and future outlook, Biomed. Pharmacother., 2021, 137, 111236.
80 B. Choi, et al., Microenvironmental regulation of stem cell behavior through biochemical and biophysical stimulation, Biomimetic Medical Materials, 2018, 147-160.

81 A. Raic, et al., Biomimetic macroporous PEG hydrogels as 3D scaffolds for the multiplication of human hematopoietic stem and progenitor cells, Biomaterials, 2014, 35(3), 929-940.

82 D. Kratzer, et al., Nanostructured bifunctional hydrogels as potential instructing platform for hematopoietic stem cell differentiation, Front. Mater., 2019, 5, 81.

83 X.-S. Jiang, et al., Surface-immobilization of adhesion peptides on substrate for ex vivo expansion of cryopreserved umbilical cord blood CD34+ cells, Biomaterials, 2006, 27(13), 2723-2732.

84 C. A. Muth, et al., Regulation of hematopoietic stem cell behavior by the nanostructured presentation of extracellular matrix components, PLoS One, 2013, 8(2), e54778.

85 A. L. Winkler, et al., Significance of nanopatterned and clustered DLL1 for hematopoietic stem cell proliferation, Adv. Funct. Mater., 2017, 27(21), 1606495.

86 N. Haque, et al., Hypoxic culture conditions as a solution for mesenchymal stem cell based regenerative therapy, $S c i$. World J., 2013, 632972.

$87 \mathrm{~K}$. Takubo, et al., Regulation of the HIF- $1 \alpha$ level is essential for hematopoietic stem cells, Cell Stem Cell, 2010, 7(3), 391-402.

88 K. Miharada, et al., Cripto regulates hematopoietic stem cells as a hypoxic-niche-related factor through cell surface receptor GRP78, Cell Stem Cell, 2011, 9(4), 330-344.

89 Y. Lu, et al., The regulation of hematopoietic stem cell fate in the context of radiation, Radiation Medicine and Protection, 2020, 1(1), 31-34.

90 A. A. Kiani, et al., Over expression of HIF-1 $\alpha$ in human mesenchymal stem cells increases their supportive functions for hematopoietic stem cells in an experimental coculture model, Hematology, 2014, 19(2), 85-98.

91 M. L. Capitano, et al., Mitigating oxygen stress enhances aged mouse hematopoietic stem cell numbers and function, J. Clin. Invest., 2021, 131(1), e140177.

92 P. Eliasson, et al., Hypoxia mediates low cell-cycle activity and increases the proportion of long-term-reconstituting hematopoietic stem cells during in vitro culture, Exp. Hematol., 2010, 38(4), 301. e2-310. e2.

$93 \mathrm{~J}$. Oswald, et al., Gene-expression profiling of CD34+ hematopoietic cells expanded in a collagen I matrix, Stem Cells, 2006, 24(3), 494-500.

94 J. C. Fontoura, et al., Comparison of 2D and 3D cell culture models for cell growth, gene expression and drug resistance, Mater. Sci. Eng., C, 2020, 107, 110264.

95 M. Darvish, et al., Umbilical cord blood mesenchymal stem cells application in hematopoietic stem cells expansion on nanofiber three-dimensional scaffold, J. Cell. Biochem., 2019, 120(7), 12018-12026.

96 F. Amirache, et al., Mystery solved: VSV-G-LVs do not allow efficient gene transfer into unstimulated $\mathrm{T}$ cells, B cells, and HSCs because they lack the LDL receptor, Blood, 2014, 123(9), 1422-1424. 
97 C.-g. Xie, et al., Marrow mesenchymal stem cells transduced with TPO/FL genes as support for ex vivo expansion of hematopoietic stem/progenitor cells, Cell. Mol. Life Sci., 2005, 62(21), 2495-2507.

98 E. J. Sitanggang, et al., Bone marrow stem cells anti-liver fibrosis potency: inhibition of hepatic stellate cells activity and extracellular matrix deposition, Int. J. Stem Cells, 2017, 10(1), 69.

99 P. Pathi, T. Ma and B. R. Locke, Role of nutrient supply on cell growth in bioreactor design for tissue engineering of hematopoietic cells, Biotechnol. Bioeng., 2005, 89(7), 743-758.

100 D. Zhou, et al., A 3D engineered scaffold for hematopoietic progenitor/stem cell co-culture in vitro, Sci. Rep., 2020, 10(1), 1-11.

101 T. Baudequin and M. Tabrizian, Multilineage Constructs for Scaffold-Based Tissue Engineering: A Review of Tissue-Specific Challenges, Adv. Healthcare Mater., 2018, 7(3), 1700734.

102 D. W. Hutmacher, M. Sittinger and M. V. Risbud, Scaffoldbased tissue engineering: rationale for computer-aided design and solid free-form fabrication systems, Trends Biotechnol., 2004, 22(7), 354-362.

103 M. Abdul-Al, et al., Stem Cell Niche Microenvironment, Bioengineering, 2021, 8(8), 108.

104 C. Chatterjee, et al., Rebuilding the hematopoietic stem cell niche: recent developments and future prospects, Acta Biomater., 2021, 132, 129-148.

105 R. Roberts, et al., Heparan sulphate bound growth factors: a mechanism for stromal cell mediated haemopoiesis, Nature, 1988, 332(6162), 376-378.

106 R. Mhanna, et al., Sulfated alginate as a mimic of sulfated glycosaminoglycans: binding of growth factors and effect on stem cell behavior, Adv. Biosyst., 2017, 1(7), 1700043.

107 C. Sun, et al., Sulfated polysaccharides interact with fibroblast growth factors and protect from denaturation, FEBS Open Bio, 2019, 9(8), 1477-1487.

$108 \emptyset$. Arlov, et al., Engineered Sulfated Polysaccharides for Biomedical Applications, Adv. Funct. Mater., 2021, 31(19), 2010732.

109 H. Kim, et al., Mesenchymal stem cell 3D encapsulation technologies for biomimetic microenvironment in tissue regeneration, Stem Cell Res. Ther., 2019, 10(1), 1-14.

$110 \mathrm{Y} . \mathrm{Xu}$, et al., Biomaterials for stem cell engineering and biomanufacturing, Bioact. Mater., 2019, 4, 366-379.

111 P. Carreras, et al., Long-term human hematopoietic stem cell culture in microdroplets, Micromachines, 2021, 12(1), 90.

112 X. Pan, et al., Encapsulated feeder cells within alginate beads for ex vivo expansion of cord blood-derived CD34+ cells, Biomater. Sci., 2016, 4(10), 1441-1453.

113 F. Amiri, et al., Co-culture of mesenchymal stem cell spheres with hematopoietic stem cells under hypoxia: a cost-effective method to maintain self-renewal and homing marker expression, Mol. Biol. Rep., 2021, 1-11.

114 J. Hur, et al., Human peripheral blood-born hematosphere as a niche for hematopoietic stem cell expansion, Cell Res., 2011, 21(6), 987-990.
115 A. O'Garra, Cytokines induce the development of functionally heterogeneous T helper cell subsets, Immunity, 1998, 8(3), 275-283.

$116 \mathrm{~J}$. Isern, et al., Self-renewing human bone marrow mesenspheres promote hematopoietic stem cell expansion, Cell Rep., 2013, 3(5), 1714-1724.

117 J. E. R. Bianco, et al., Characterization of a novel decellularized bone marrow scaffold as an inductive environment for hematopoietic stem cells, Biomater. Sci., 2019, $7(4), 1516-1528$.

118 Y. Hashimoto, et al., The effect of decellularized bone/bone marrow produced by high-hydrostatic pressurization on the osteogenic differentiation of mesenchymal stem cells, Biomaterials, 2011, 32(29), 7060-7067.

119 N. Nakamura, et al., Induction of in vivo ectopic hematopoiesis by a three-dimensional structured extracellular matrix derived from decellularized cancellous bone, ACS Biomater. Sci. Eng., 2019, 5(11), 5669-5680.

120 M. Kräter, et al., Bone marrow niche-mimetics modulate HSPC function via integrin signaling, Sci. Rep., 2017, 7(1), 1-15.

121 N. Soffer-Tsur, D. Peer and T. Dvir, ECM-based macroporous sponges release essential factors to support the growth of hematopoietic cells, J. Controlled Release, 2017, 257, 84-90.

122 D. Li, et al., Decellularized Wharton jelly matrix: a biomimetic scaffold for ex vivo hematopoietic stem cell culture, Blood Adv., 2019, 3(7), 1011-1026.

123 A. Lavrentieva, et al., Effects of hypoxic culture conditions on umbilical cord-derived human mesenchymal stem cells, Cell Commun. Signaling, 2010, 8(1), 1-9.

124 Y. P. Han, Matrix metalloproteinases, the pros and cons, in liver fibrosis, J. Gastroenterol. Hepatol., 2006, 21, S88-S91.

125 S. Clara-Trujillo, et al., Biomimetic microspheres for 3D mesenchymal stem cell culture and characterization, Colloids Surf., B, 2019, 177, 68-76.

126 Y. Cai, et al., Porous microsphere and its applications, Int. J. Nanomed., 2013, 8, 1111.

127 C. D. Liu, X. F. Tu and F. Chen, Neovascularization by Sustained Delivery of G-CSF, EPO and VEGF Using Dextran/ PLGA Microspheres, Ann. Vasc. Surg., 2020, 64, 328-338.

128 B. P. Mahadik, et al., Microfluidic generation of gradient hydrogels to modulate hematopoietic stem cell culture environment, Adv. Healthcare Mater., 2014, 3(3), 449-458.

129 C. Wang, et al., Microsphere-structured hydrogel crosslinked by polymerizable protein-based nanospheres, Polymer, 2020, 211, 123114.

130 K. M. Z. Hossain, U. Patel and I. Ahmed, Development of microspheres for biomedical applications: a review, Prog. Biomater., 2015, 4(1), 1-19.

131 C. Bello-Rodriguez, et al., A 3D construct based on mesenchymal stromal cells, collagen microspheres and plasma clot supports the survival, proliferation and differentiation of hematopoietic cells in vivo, Cell Tissue Res., 2020, 382(3), 499-507.

132 J. E. Nichols, et al., In vitro analog of human bone marrow from 3D scaffolds with biomimetic inverted colloidal crystal geometry, Biomaterials, 2009, 30(6), 1071-1079. 
133 E. Fathi, R. Farahzadi and B. Valipour, Alginate/gelatin encapsulation promotes NK cells differentiation potential of bone marrow resident C-kit+ hematopoietic stem cells, Int. J. Biol. Macromol., 2021, 177, 317-327.

134 J. Zhong, et al., Formation of novel mesoporous TiC microspheres through a sol-gel and carbothermal reduction process, J. Eur. Ceram. Soc., 2012, 32(12), 3407-3414.

$135 \mathrm{~J} . \mathrm{Li}$, et al., The Potential of electrospinning/electrospraying technology in the rational design of hydrogel structures, Macromol. Mater. Eng., 2020, 305(8), 2000285.

136 N. Annabi, et al., Controlling the porosity and microarchitecture of hydrogels for tissue engineering, Tissue Eng., Part B, 2010, 16(4), 371-383.

137 Y. Gong, et al., Microcavitary hydrogel-mediating phase transfer cell culture for cartilage tissue engineering, Tissue Eng., Part A, 2010, 16(12), 3611-3622.

138 P. T. Thevenot, et al., The effect of incorporation of SDF- $1 \alpha$ into PLGA scaffolds on stem cell recruitment and the inflammatory response, Biomaterials, 2010, 31(14), 3997-4008.

139 X. Pan, et al., Biomimetic macroporous pcl scaffolds for ex vivo expansion of cord blood-derived CD34+ cells with feeder cells support, Macromol. Biosci., 2017, $17(8), 1700054$.

140 M. S. V. Ferreira, et al., Cord blood-hematopoietic stem cell expansion in 3D fibrin scaffolds with stromal support, Biomaterials, 2012, 33(29), 6987-6997.

141 G. H. Yang, et al., Investigating the physical characteristics and cellular interplay on 3D-printed scaffolds depending on the incorporated silica size for hard tissue regeneration, Mater. Des., 2021, 109866.

142 T. Subbiah, et al., Electrospinning of nanofibers, J. Appl. Polym. Sci., 2005, 96(2), 557-569.

143 D. H. Reneker and A. L. Yarin, Electrospinning jets and polymer nanofibers, Polymer, 2008, 49(10), 2387-2425.

144 M. S. V. Ferreira and S. H. Mousavi, Nanofiber technology in the ex vivo expansion of cord blood-derived hematopoietic stem cells, Nanomedicine, 2018, 14(5), 1707-1718.

145 H. Das, et al., Ex vivo nanofiber expansion and genetic modification of human cord blood-derived progenitor/ stem cells enhances vasculogenesis, Cell Transplant., 2009, 18(3), 305-318.

146 K.-N. Chua, et al., Surface-aminated electrospun nanofibers enhance adhesion and expansion of human umbilical cord blood hematopoietic stem/progenitor cells, Biomaterials, 2006, 27(36), 6043-6051.

147 F. Sabaghi, et al., Evaluation of human cord blood CD34+ hematopoietic stem cell differentiation to megakaryocyte on aminated PES nanofiber scaffold compare to 2-D culture system, Artif. Cells, Nanomed., Biotechnol., 2016, 44(4), 1062-1068.

148 K.-N. Chua, et al., Functional nanofiber scaffolds with different spacers modulate adhesion and expansion of cryopreserved umbilical cord blood hematopoietic stem/ progenitor cells, Exp. Hematol., 2007, 35(5), 771-781.

$149 \mathrm{~J}$. Cai, et al., Bone marrow stem cells-seeded polyethylene terephthalate scaffold in repair and regeneration of Rabbit Achilles Tendon, Artif. Organs, 2018, 42(11), 1086-1094.
150 Q. Feng, et al., Expansion of engrafting human hematopoietic stem/progenitor cells in three-dimensional scaffolds with surface-immobilized fibronectin, J. Biomed. Mater. Res., Part A, 2006, 78(4), 781-791.

151 C. E. Severn, et al., Polyurethane scaffolds seeded with CD34+ cells maintain early stem cells whilst also facilitating prolonged egress of haematopoietic progenitors, Sci. Rep., 2016, 6(1), 1-12.

152 C. Severn, et al., Ex vivo culture of adult CD34+ stem cells using functional highly porous polymer scaffolds to establish biomimicry of the bone marrow niche, Biomaterials, 2019, 225, 119533.

153 T. Bai, et al., Expansion of primitive human hematopoietic stem cells by culture in a zwitterionic hydrogel, Nat. Med., 2019, 25(10), 1566-1575.

154 C. H. Cho, J. F. Eliason and H. W. Matthew, Application of porous glycosaminoglycan-based scaffolds for expansion of human cord blood stem cells in perfusion culture, J. Biomed. Mater. Res., Part A, 2008, 86(1), 98-107.

155 R. W. Nurhayati, et al., Development of double-layered alginate-chitosan hydrogels for human stem cell microencapsulation. in AIP Conference Proceedings, AIP Publishing LLC, 2019.

156 V. Keskar, et al., In vitro evaluation of macroporous hydrogels to facilitate stem cell infiltration, growth, and mineralization, Tissue Eng., Part A, 2009, 15(7), 1695-1707.

157 Y. Ling, et al., A cell-laden microfluidic hydrogel, Lab Chip, 2007, 7(6), 756-762.

158 J. S. Miller, et al., Rapid casting of patterned vascular networks for perfusable engineered three-dimensional tissues, Nat. Mater., 2012, 11(9), 768-774.

159 S. J. Bryant, et al., Photo-patterning of porous hydrogels for tissue engineering, Biomaterials, 2007, 28(19), 2978-2986.

160 O. Sarig-Nadir, et al., Laser photoablation of guidance microchannels into hydrogels directs cell growth in three dimensions, Biophys. J., 2009, 96(11), 4743-4752.

161 M. T. A. P. Kresnowati, G. Forde and X. D. Chen, Modelbased analysis and optimization of bioreactor for hematopoietic stem cell cultivation, Bioprocess Biosyst. Eng., 2011, 34(1), 81-93.

162 G.-J. Wang, et al., Fabrication of PLGA microvessel scaffolds with circular microchannels using soft lithography, J. Micromech. Microeng., 2007, 17(10), 2000.

163 H.-W. Wu, et al., An integrated microfluidic system for isolation, counting, and sorting of hematopoietic stem cells, Biomicrofluidics, 2010, 4(2), 024112.

164 S. L. Faley, et al., Microfluidic single cell arrays to interrogate signalling dynamics of individual, patient-derived hematopoietic stem cells, Lab Chip, 2009, 9(18), 2659-2664.

165 X. Han, et al., Microfluidic cell trap arrays for single hematopoietic stem/progenitor cell behavior analysis, Proteomics, 2020, 20(13), 1900223.

166 Y.-s. Torisawa, et al., Bone marrow-on-a-chip replicates hematopoietic niche physiology in vitro, Nat. Methods, 2014, 11(6), 663-669. 
167 S. Sieber, et al., Bone marrow-on-a-chip: long-term culture of human haematopoietic stem cells in a threedimensional microfluidic environment, J. Tissue Eng. Regener. Med., 2018, 12(2), 479-489.

168 U. Jammalamadaka and K. Tappa, Recent advances in biomaterials for 3D printing and tissue engineering, J. Funct. Biomater., 2018, 9(1), 22.

169 T. Distler and A. R. Boccaccini, 3D printing of electrically conductive hydrogels for tissue engineering and biosensors-A review, Acta Biomater., 2020, 101, 1-13.

$170 \mathrm{~W}$. Zhu, et al., 3D printing of functional biomaterials for tissue engineering, Curr. Opin. Biotechnol, 2016, 40, 103-112.

171 T.-S. Jang, et al., 3D printing of hydrogel composite systems: Recent advances in technology for tissue engineering, Int. J. Bioprint., 2018, 4(1), 126.

172 F. Pati, et al., Printing three-dimensional tissue analogues with decellularized extracellular matrix bioink, Nat. Commun., 2014, 5(1), 1-11.

173 C. Marques, et al., Collagen-based bioinks for hard tissue engineering applications: a comprehensive review, J. Mater. Sci.: Mater. Med., 2019, 30(3), 1-12.

174 J. D. Kim, et al., Piezoelectric inkjet printing of polymers: Stem cell patterning on polymer substrates, Polymer, 2010, 51(10), 2147-2154.

175 H. Hong, et al., Digital light processing 3D printed silk fibroin hydrogel for cartilage tissue engineering, Biomaterials, 2020, 232, 119679.

176 Y.-L. Cheng and F. Chen, Preparation and characterization of photocured poly ( $\varepsilon$-caprolactone) diacrylate/poly (ethylene glycol) diacrylate/chitosan for photopolymerizationtype 3D printing tissue engineering scaffold application, Mater. Sci. Eng., C, 2017, 81, 66-73.

177 P. Soman, et al., Digital microfabrication of user-defined 3D microstructures in cell-laden hydrogels, Biotechnol. Bioeng., 2013, 110(11), 3038-3047.

178 L. Koch, et al., Laser assisted cell printing, Curr. Pharm. Biotechnol., 2013, 14(1), 91-97.

179 J. Zhang, et al., Digital light processing based threedimensional printing for medical applications, Int. J. Bioprint., 2020, 6(1), 242.

180 S. Radhakrishnan, et al., Fabrication of 3D printed antimicrobial polycaprolactone scaffolds for tissue engineering applications, Mater. Sci. Eng., C, 2021, 118, 111525.

181 P. Mikes, et al., Comparison and characterization of different polyester nano/micro fibres for use in tissue engineering applications, J. Ind. Text., 2021, 50(6), 870-890.

182 G. S. Georgiev, et al., Self-assembly, antipolyelectrolyte effect, and nonbiofouling properties of polyzwitterions, Biomacromolecules, 2006, 7(4), 1329-1334.

183 J. Maitra and V. K. Shukla, Cross-linking in hydrogels-a review, Am. J. Polym. Sci., 2014, 4(2), 25-31.

184 L. Rebers, et al., Differentiation of physical and chemical cross-linking in gelatin methacryloyl hydrogels, Sci. Rep., 2021, 11(1), 1-12.

185 B.-S. Kim, C. E. Baez and A. Atala, Biomaterials for tissue engineering, World J. Urol., 2000, 18(1), 2-9.
186 R. Mohammadinejad, et al., Recent advances in natural gum-based biomaterials for tissue engineering and regenerative medicine: a review, Polymers, 2020, 12(1), 176.

187 Y. Yuan, et al., Novel alginate three-dimensional static and rotating culture systems for effective ex vivo amplification of human cord blood hematopoietic stem cells and in vivo functional analysis of amplified cells in NOD/SCID mice, Transfusion, 2013, 53(9), 2001-2011.

188 C. A. Moore, et al., A 3d bioprinted material that recapitulates the perivascular bone marrow structure for sustained hematopoietic and cancer models, Polymers, 2021, 13(4), 480.

189 M. V. Braham, et al., A human hematopoietic niche model supporting hematopoietic stem and progenitor cells in vitro, Adv. Healthcare Mater., 2019, 8(10), 1801444.

190 C. Brieke, et al., Light-controlled tools, Angew. Chem., Int. Ed., 2012, 51(34), 8446-8476.

191 A. E. Gilchrist and B. A. Harley, Connecting secretome to hematopoietic stem cell phenotype shifts in an engineered bone marrow niche, Integr. Biol., 2020, 12(7), 175-187.

192 A. E. Gilchrist, et al., Soluble Signals and Remodeling in a Synthetic Gelatin-Based Hematopoietic Stem Cell Niche, Adv. Healthcare Mater., 2019, 8(20), 1900751.

193 Y. Zhang, et al., Promoted proliferation of hematopoietic stem cells enabled by a hyaluronic acid/carbon nanotubes antioxidant hydrogel, Macromol. Mater. Eng., 2019, 304(4), 1800630.

194 M. Xie, et al., Facile 3D cell culture protocol based on photocurable hydrogels, Bio-Des. Manuf., 2021, 4(1), 149-153.

195 A. E. Gilchrist, et al., Encapsulation of murine hematopoietic stem and progenitor cells in a thiol-crosslinked maleimide-functionalized gelatin hydrogel, Acta Biomater., 2021, 131, 138-148.

196 V. Jayawarna, et al., Nanostructured hydrogels for threedimensional cell culture through self-assembly of fluorenylmethoxycarbonyl-dipeptides, Adv. Mater., 2006, 18(5), 611-614.

197 M. J. Webber and E. T. Pashuck, Macro) molecular selfassembly for hydrogel drug delivery, Adv. Drug Delivery Rev., 2021, 172, 275-295.

198 I. Leisten, et al., 3D co-culture of hematopoietic stem and progenitor cells and mesenchymal stem cells in collagen scaffolds as a model of the hematopoietic niche, Biomaterials, 2012, 33(6), 1736-1747.

199 S. Mousavi, et al., Potential of Polycaprolactone Nanofiber Scaffold for Ex Vivo Expansion of Cord Blood-Derived CD34+ Hematopoietic Stem Cells, Int. J. Stem Cell Res. Ther., 2019, 5, 059.

200 A. C. Brown and T. H. Barker, Fibrin-based biomaterials: modulation of macroscopic properties through rational design at the molecular level, Acta Biomater., 2014, 10(4), 1502-1514.

201 C. H. Park and K. M. Woo, Fibrin-based biomaterial applications in tissue engineering and regenerative medicine, Biomimetic Medical Materials, 2018, 253-261. 
202 C. Garcia-Abrego, et al., Towards mimicking the fetal liver niche: the influence of elasticity and oxygen tension on hematopoietic stem/progenitor cells cultured in 3D fibrin hydrogels, Int. J. Mol. Sci., 2020, 21(17), 6367.

203 M. Islami, et al., In vitro expansion of CD 133+ cells derived from umbilical cord blood in poly-L-lactic acid (PLLA) scaffold coated with fibronectin and collagen, Artif. Cells, Nanomed., Biotechnol., 2018, 46(5), 1025-1033.

204 F. Eskandari, et al., Nanofiber expansion of umbilical cord blood hematopoietic stem cells, Iran. J. Pediatr. Hematol. Oncol., 2015, 5(4), 170.
205 X. Jiang, et al., Endometrial membrane organoids from human embryonic stem cell combined with the 3D Matrigel for endometrium regeneration in asherman syndrome, Bioact. Mater., 2021, 6(11), 3935-3946.

206 Z. Yang, H. Xu and X. Zhao, Designer self-assembling peptide hydrogels to engineer $3 \mathrm{D}$ cell microenvironments for cell constructs formation and precise oncology remodeling in ovarian cancer, Adv. Sci., 2020, 7(9), 1903718.

207 C. Karavasili and D. G. Fatouros, Self-assembling peptides as vectors for local drug delivery and tissue engineering applications, Adv. Drug Delivery Rev., 2021, 174, 387-405. 